Article

\title{
New Steroidal Saponins Isolated from the Rhizomes of Paris mairei
}

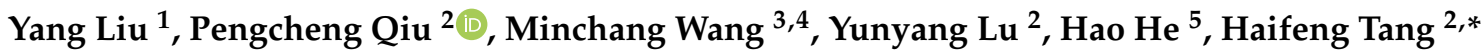 \\ and Bang-Le Zhang ${ }^{1, *}$
}

1 Department of Pharmaceutics, School of Pharmacy, Air Force Medical University, Xi'an 710032, China; so870823@163.com

2 Department of Chinese Materia Medica and Natural Medicines, School of Pharmacy, Air Force Medical University, Xi'an 710032, China; qpc023@126.com (P.Q.); luyunyanggq@163.com (Y.L.)

3 Xi'an Modern Chemistry Research Institute, X' ${ }^{\prime}$ an 710065, China; wmc204@163.com

4 State Key Laboratory of Fluorine \& Nitrogen Chemicals, Xi'an 710065, China

5 School of Pharmacy, Xi'an Medical University, Xi'an 710021, China; hehao313@163.com

* Correspondence: tanghf71@fmmu.edu.cn (H.T.); blezhang@fmmu.edu.cn (B.-L.Z.); Tel.: +86-029-8477-4748 (H.T.); +86-029-8471-1513 (B.-L.Z.)

check for updates

Citation: Liu, Y.; Qiu, P.; Wang, M.; Lu, Y.; He, H.; Tang, H.; Zhang, B.-L. New Steroidal Saponins Isolated from the Rhizomes of Paris mairei. Molecules 2021, 26, 6366. https:// doi.org/10.3390/molecules26216366

Academic Editors: John A. Beutler and Luisella Verotta

Received: 22 September 2021

Accepted: 18 October 2021

Published: 21 October 2021

Publisher's Note: MDPI stays neutral with regard to jurisdictional claims in published maps and institutional affiliations.

Copyright: (C) 2021 by the authors Licensee MDPI, Basel, Switzerland. This article is an open access article distributed under the terms and conditions of the Creative Commons Attribution (CC BY) license (https:// creativecommons.org/licenses/by/ $4.0 /)$.

\begin{abstract}
The genus Paris is an excellent source of steroidal saponins that exhibit various bioactivities. Paris mairei is a unique species and has been widely used as folk medicine in Southwest China for a long time. With the help of chemical methods and modern spectra analysis, five new steroidal saponins, pamaiosides A-E (1-5), along with five known steroidal saponins 6-10, were isolated from the rhizomes of Paris mairei. The cytotoxicity of all the new saponins was evaluated against human pancreatic adenocarcinoma PANC-1 and BxPC3 cell lines.
\end{abstract}

Keywords: Paris mairei; steroidal saponins; spirostane saponin; 15-oxo-18-nor-spirost; cytotoxicity

\section{Introduction}

The genus Paris (Liliaceae) includes 33 species around the world, and 27 species and more than 15 varieties have been discovered in China [1]. It has been used as a traditional Chinese medicine for traumatic injuries, heat-clearing and detoxifying, and relief of swelling and long-term pain [2]. Under the development of phytochemistry, steroidal saponins have been proved to be the main chemicals in the genus Paris and present a wide range of pharmacological activities such as anti-tumor [3-5], anti-inflammatory [6], anti-fungal [7], hemostasis [8], and immunomodulatory [9]. Moreover, Rhizoma Paridis, documented as rhizomes of Paris polyphylla var. yannanensis and Paris polyphylla var. Chinensis in the 2020 edition of the Chinese Pharmacopoeia, is usually used as adjuvant drugs for postoperative treatment of cancer to improve symptoms and therapeutic effect. However, Paris polyphylla var. yannanensis and Paris polyphylla var. Chinensis as perennial plants need at least 5 years to mature, and the increasing market demand makes wild sources of Rhizoma Paridis seriously scarce [10]. Hence, it is necessary to investigate other species of Paris in order to relieve resource pressure. Paris mairei is mainly distributed in the Guizhou, Sichuan, and Yunnan provinces of China and used as folk medicine for a long time. Herein, this paper reports the isolation and structural identification of five new (1-5) and five known (6-10) saponins (Figure 1) as well as the cytotoxicity against human pancreatic adenocarcinoma PANC-1 and BxPC3 cell lines. 

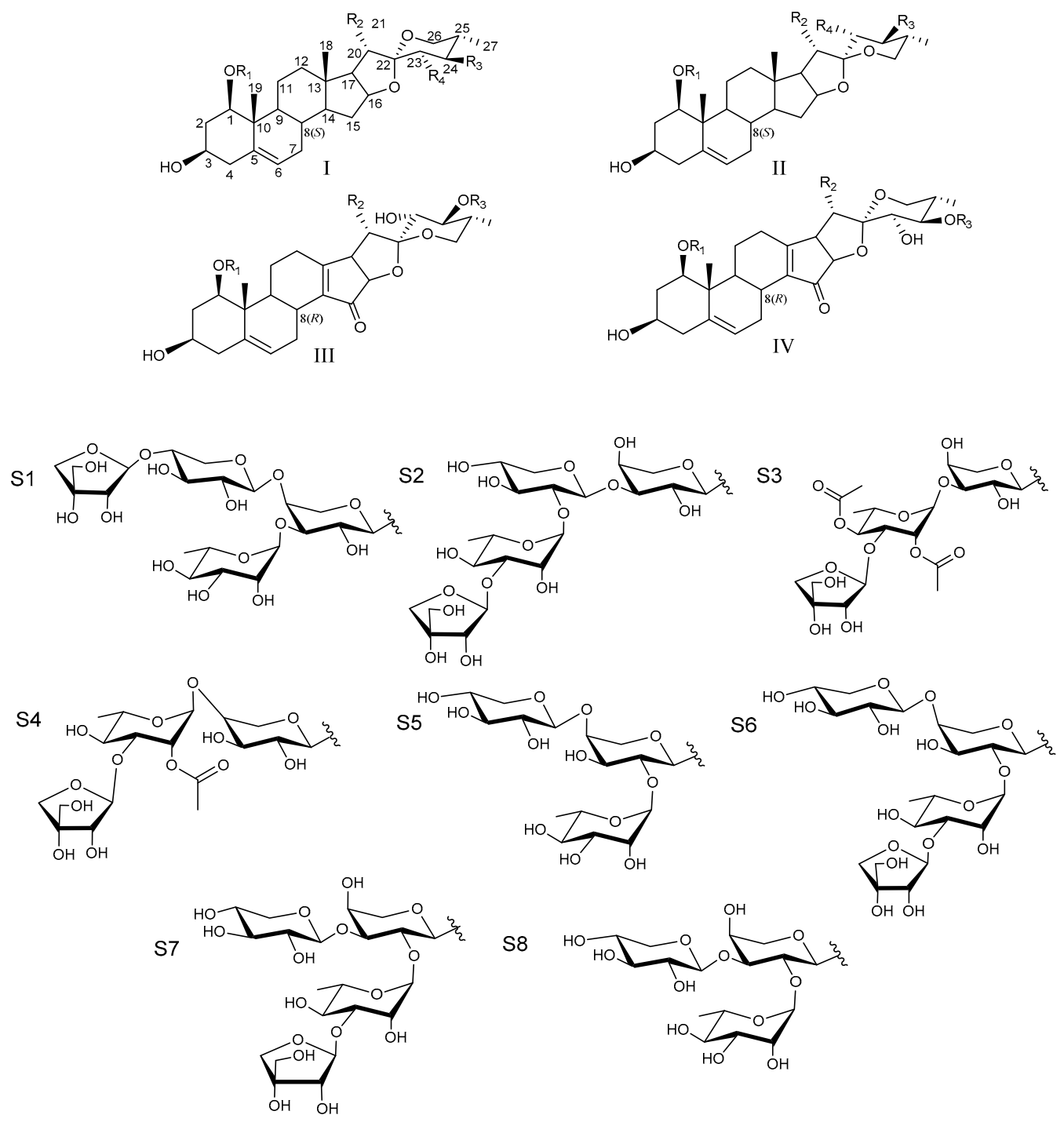

\begin{tabular}{cccccc}
\hline Compound & Aglycone & $\mathbf{R}_{\mathbf{1}}$ & $\mathbf{R}_{2}$ & $\mathbf{R}_{3}$ & $\mathbf{R}_{4}$ \\
\hline $\mathbf{1}$ & I & $\mathrm{S}_{1}$ & $\mathrm{CH}_{3}$ & $\mathrm{H}$ & $\mathrm{H}$ \\
$\mathbf{2}$ & II & $\mathrm{S}_{2}$ & $\mathrm{CH}_{2} \mathrm{OH}$ & $\mathrm{OH}$ & $\mathrm{OH}$ \\
$\mathbf{3}$ & III & $\mathrm{S}_{3}$ & $\mathrm{CH}_{2} \mathrm{OH}$ & $\mathrm{H}$ & - \\
$\mathbf{4}$ & III & $\mathrm{S}_{4}$ & $\mathrm{CH}_{2} \mathrm{OCOCH}_{3}$ & $\mathrm{H}$ & - \\
$\mathbf{5}$ & IV & $\mathrm{S}_{5}$ & $\mathrm{CH}_{3}$ & $\mathrm{COCH}_{3}$ & - \\
$\mathbf{6}$ & I & $\mathrm{S}_{6}$ & $\mathrm{CH}_{2} \mathrm{OH}$ & $\mathrm{OH}$ & $\mathrm{OH}$ \\
$\mathbf{7}$ & II & $\mathrm{S}_{7}$ & $\mathrm{CH}_{2} \mathrm{OH}$ & $\mathrm{H}$ & - \\
$\mathbf{8}$ & II & $\mathrm{S}_{7}$ & $\mathrm{CH}_{3}$ & $\mathrm{COCH}$ & - \\
$\mathbf{9}$ & II & $\mathrm{S}_{6}$ & $\mathrm{CH}_{2} \mathrm{OH}$ & $\mathrm{H}$ & - \\
$\mathbf{1 0}$ & I & $\mathrm{S}_{8}$ & $\mathrm{CH}_{2} \mathrm{OH}$ & $\mathrm{OH}$ & $\mathrm{OH}$ \\
\hline
\end{tabular}

Figure 1. Structures of compounds 1-10.

\section{Results and Discussion}

Compound 1, named Pamaiosides A, a white amorphous solid, was positive to Liebermann Burchard and Molisch chemical reactions, which indicates that it might be a steroidal glycoside. The pseudomolecular ion peak was detected in the HR-ESI-MS spectrum at $m / z 995.4824[\mathrm{M}+\mathrm{Na}]^{+}$(calculated for $\mathrm{C}_{48} \mathrm{H}_{76} \mathrm{O}_{20} \mathrm{Na}$, 995.4828), corresponding to the molecular formula $\mathrm{C}_{48} \mathrm{H}_{76} \mathrm{O}_{20}$. Four methyl groups were tested in the ${ }^{1} \mathrm{H}-\mathrm{NMR}$ 
spectrum at $\delta_{\mathrm{H}} 0.82(3 \mathrm{H}, s, \mathrm{H}-18), 1.12(3 \mathrm{H}, s, \mathrm{H}-19), \delta_{\mathrm{H}} 0.80(3 \mathrm{H}, d, J=6.35 \mathrm{~Hz}, \mathrm{H}-27)$, and $0.96(3 \mathrm{H}, d, J=6.90 \mathrm{~Hz}, \mathrm{H}-21)$. Meanwhile, one olefinic methine proton signal was observed at $\delta_{\mathrm{H}} 5.56(1 \mathrm{H}$, br s, H-6). The hydrogen signals above suggest a steroid skeleton $[11,12]$. Correspondingly, in the ${ }^{13} \mathrm{C}-\mathrm{NMR}$ spectrum of $\mathbf{1}$, four carbon signals of methyl groups were revealed at $\delta_{\mathrm{C}} 17.65$ (C-18), 15.52 (C-19), 17.29 (C-27), and 15.07 (C-21) as well as one trisubstituted double bonds at $\delta_{\mathrm{C}} 139.71$ (C-5) and 126.17 (C-6). A characteristic hemiacetal signal of spirostanol aglycone was discovered at $\delta_{\mathrm{C}} 111.74(\mathrm{C}-22)$ [11]. In the HMBC spectrum, the cross-peaks between $\mathrm{H}-4\left(\delta_{\mathrm{H}} 1.90\right)$ and $\mathrm{C}-5\left(\delta_{\mathrm{C}} 139.71\right), \mathrm{H}-19\left(\delta_{\mathrm{H}} 1.12\right)$ and $\mathrm{C}-5\left(\delta_{\mathrm{C}} 139.71\right)$, and $\mathrm{H}-6\left(\delta_{\mathrm{H}} 5.56\right)$ and $\mathrm{C}-8\left(\delta_{\mathrm{C}} 34.26\right) / \mathrm{C}-10\left(\delta_{\mathrm{C}} 43.58\right)$ inferred that the double bond was located at C-5/C-6 (Figure 2). In the NOESY spectrum, the correlation between $\mathrm{H}-1\left(\delta_{\mathrm{H}} 3.37\right)$ and $\mathrm{H}-9\left(\delta_{\mathrm{H}} 1.25\right)$ and $\mathrm{H}-3\left(\delta_{\mathrm{H}} 3.34\right)$ and H-9 $\left(\delta_{\mathrm{H}} 1.25\right)$ suggested that the configurations of $\mathrm{H}-1$ and $\mathrm{H}-3$ were an $\alpha$-orientation, so that the hydroxyl substituent at C-1 and C-3 were both $\beta$ configuration. The correlation between $\mathrm{H}-3\left(\delta_{\mathrm{H}} 3.34\right)$ and $\mathrm{H}-16\left(\delta_{\mathrm{H}} 4.38\right) / \mathrm{H}-17\left(\delta_{\mathrm{H}} 1.72\right)$, between $\mathrm{H}-16\left(\delta_{\mathrm{H}} 4.38\right)$ and $\mathrm{H}-17\left(\delta_{\mathrm{H}} 1.72\right)$, between $\mathrm{H}-8\left(\delta_{\mathrm{H}} 1.56\right)$ and $\mathrm{H}-18\left(\delta_{\mathrm{H}} 0.82\right)$, between $\mathrm{H}-19\left(\delta_{\mathrm{H}} 1.12\right)$ and $\mathrm{H}-11\left(\delta_{\mathrm{H}} 1.42\right)$, and between $\mathrm{H}-9\left(\delta_{\mathrm{H}} 1.25\right)$ and $\mathrm{H}-14\left(\delta_{\mathrm{H}} 1.15\right)$ elucidate the usual trans junction for the $\mathrm{B} / \mathrm{C}$ and $\mathrm{C} / \mathrm{D}$ rings. The correlations between $\mathrm{H}-8\left(\delta_{\mathrm{H}} 1.56\right)$ and $\mathrm{H}-20\left(\delta_{\mathrm{H}} 1.90\right)$ infer that C-20 was an $S$ configuration. In the spirostanol saponins, when the resonance of the proton $\mathrm{H}-20$ was observed at a lower field than approximately $\delta_{\mathrm{H}} 2.48$, the orientation relationship between the proton of $\mathrm{H}-20$ and the oxygen atom included in the F ring was thought to be located at the cis position. On the other hand, when the proton shifts of $\mathrm{H}-20$ were detected at a higher field than $\delta_{\mathrm{H}} 2.20$, the orientation relationship is thought to be trans $[13,14]$. In this way, the orientation relationship of the F ring was considered to be trans, and the configuration of C-22 was confirmed as $R$. The $25 R$ configuration was determined by the chemical shift difference between H-26a and H-26b $\left(\Delta=\delta_{\mathrm{Ha}}-\delta_{\mathrm{Hb}}=3.43-3.30=0.13<0.48\right)[15,16]$. By combining the data and consulting the literature [17], the aglycone of compound 1 was identified as $(20 S, 22 R, 25 R)$-spirost-5-en- $1 \beta, 3 \beta$-diol.

According to the ${ }^{13} \mathrm{C}-\mathrm{NMR}$ spectrum, except for the 27 signals of aglycone, the remaining 21 belonged to the oligosaccharide's moiety. After acid hydrolysis and derivatization with N(trimethylsilyl) imidazole, the derivates were compared with retention times to the corresponding authentic samples by GC analysis; thus, the monosaccharide residues were identified as L-Ara, L-Rha, D-Xyl, and D-Api in a ratio of 1:1:1:1. In the ${ }^{1} \mathrm{H}-\mathrm{NMR}$ spectrum, four anomeric proton signals were obvious at $\delta_{\mathrm{H}} 4.34\left(d, J=7.35 \mathrm{~Hz}, \mathrm{H}-1\right.$ of Ara), $\delta_{\mathrm{H}} 5.31$ (br s, H-1 of Rha), $\delta_{\mathrm{H}} 4.41(b \mathrm{r} d, J=7.1 \mathrm{~Hz}, \mathrm{H}-1$ of $\mathrm{Xyl})$, and $\delta_{\mathrm{H}} 5.19(d, J=2.9 \mathrm{~Hz}, \mathrm{H}-1$ of Api). The corresponding carbon signals were successfully searched at $\delta_{\mathrm{C}} 101.16, \delta_{\mathrm{C}} 101.60, \delta_{\mathrm{C}} 106.47$, and $\delta_{\mathrm{C}} 112.17$ in the HSQC spectrum, respectively. By analyzing the ${ }^{1} \mathrm{H}-\mathrm{NMR}, \mathrm{TOCSY}$, and HSQC spectra, the sequence and location of protons and carbons were determined in each monosaccharide (Tables 1-4). The sequence of a tetrasaccharide chain was confirmed by the HMBC spectrum, which acted as the correlations from Rha H-1 $\left(\delta_{\mathrm{H}} 5.31\right)$ to Ara C-3 $\left(\delta_{\mathrm{C}} 80.45\right)$, Api $\mathrm{H}-1\left(\delta_{\mathrm{H}} 5.19\right)$ to $\mathrm{Xyl} \mathrm{C}-4\left(\delta_{\mathrm{C}} 70.54\right), \mathrm{Xyl} \mathrm{H}-1\left(\delta_{\mathrm{H}} 4.41\right)$ to Ara C-4 $\left(\delta_{\mathrm{C}} 85.29\right)$, and the crosspeak between Ara H-1 $\left(\delta_{\mathrm{H}} 4.34\right)$ and $\mathrm{C}-1\left(\delta_{\mathrm{C}} 84.79\right)$ demonstrated the location of a sugar linkage. The anomeric proton coupling constants of D-xylopyranose $(J=7.1 \mathrm{~Hz}>7.0 \mathrm{~Hz})$ and L-arabopyranose $(J=7.35 \mathrm{~Hz}>7.0 \mathrm{~Hz})$ suggested that the configurations had a $\beta$-orientation and an $\alpha$-orientation, respectively $[18,19]$. The $\beta$ configuration of $\mathrm{D}$-apiose was determined by the chemical shifts of $\delta_{\mathrm{C}} 112.17(\mathrm{C}-1), \delta_{\mathrm{C}} 78.23(\mathrm{C}-2), \delta_{\mathrm{C}} 80.49(\mathrm{C}-3), \delta_{\mathrm{C}} 75.18(\mathrm{C}-4)$, and $\delta_{\mathrm{C}} 65.56$ (C-5) [20]; the $\alpha$ anomeric configuration of L-rhamnopyranosyl was confirmed by the chemical shifts of Rha C-5 at $\delta_{C} 69.84$ [21]. Thus, the structure of Pamaiosides A (1) was characterized as $(20 S, 22 R, 25 R)$-spirost-5-en- $\beta, 3 \beta$-diol-1-O- $\beta$-D-apiofuranosyl- $(1 \rightarrow 4)-\beta$-Dxylopyranosyl-( $1 \rightarrow 4)$-[ $\alpha$-L-rhamnopyranosyl- $(1 \rightarrow 3)]$ - $a$-L-arabinopyranoside. 
Table 1. ${ }^{13} \mathrm{C}-\mathrm{NMR}$ data of aglycone moieties for compounds $\mathbf{1 - 5}$ in $\mathrm{CD}_{3} \mathrm{OD}$.

\begin{tabular}{|c|c|c|c|c|c|}
\hline \multirow{2}{*}{ Number } & \multicolumn{5}{|c|}{ Compounds $\left(\delta_{\mathrm{C}}\right)$} \\
\hline & $1^{a}$ & $2^{a}$ & $3^{b}$ & $4^{b}$ & $5^{a}$ \\
\hline 1 & 84.79 & 84.80 & 85.28 & 85.33 & 85.57 \\
\hline 2 & 37.45 & 37.46 & 37.88 & 37.66 & 37.55 \\
\hline 3 & 69.37 & 69.33 & 69.19 & 69.37 & 69.34 \\
\hline 4 & 43.52 & 43.52 & 42.80 & 42.91 & 43.04 \\
\hline 5 & 139.71 & 139.79 & 139.82 & 139.69 & 139.70 \\
\hline 6 & 126.17 & 126.12 & 126.42 & 126.19 & 126.15 \\
\hline 7 & 33.04 & 32.86 & 30.32 & 30.27 & 30.27 \\
\hline 8 & 34.26 & 34.27 & 32.88 & 32.93 & 32.79 \\
\hline 9 & 51.54 & 51.53 & 48.91 & 48.86 & 48.76 \\
\hline 10 & 43.58 & 43.61 & 43.36 & 43.30 & 43.31 \\
\hline 11 & 24.95 & 24.84 & 26.24 & 26.27 & 21.27 \\
\hline 12 & 41.40 & 41.24 & 29.17 & 29.09 & 29.28 \\
\hline 13 & 41.29 & 41.80 & 179.23 & 178.36 & 179.36 \\
\hline 14 & 58.11 & 58.26 & 139.51 & 140.38 & 139.58 \\
\hline 15 & 32.85 & 33.13 & 207.09 & 206.45 & 207.11 \\
\hline 16 & 82.38 & 84.65 & 82.38 & 82.19 & 82.92 \\
\hline 17 & 64.14 & 58.63 & 49.66 & 49.84 & 52.38 \\
\hline 18 & 17.65 & 17.30 & - & - & - \\
\hline 19 & 15.52 & 15.53 & 14.35 & 14.31 & 14.38 \\
\hline 20 & 43.05 & 46.04 & 49.54 & 46.32 & 43.66 \\
\hline 21 & 15.07 & 62.95 & 61.99 & 64.53 & 14.08 \\
\hline 22 & 110.74 & 112.72 & 114.60 & 114.25 & 113.48 \\
\hline 23 & 32.59 & 71.20 & 74.51 & 74.48 & 67.53 \\
\hline 24 & 30.04 & 74.01 & 76.27 & 76.10 & 73.87 \\
\hline 25 & 31.59 & 36.57 & 39.33 & 39.47 & 35.39 \\
\hline 26 & 67.97 & 61.39 & 65.81 & 65.90 & 62.38 \\
\hline \multirow[t]{2}{*}{27} & 17.29 & 12.97 & 13.29 & 13.25 & 12.52 \\
\hline & & & & 21-O-acetyl & 24-O-acetyl \\
\hline 1 & - & - & - & 172.90 & 173.78 \\
\hline 2 & - & - & - & 21.12 & 21.27 \\
\hline
\end{tabular}

a Tested in ${ }^{13} \mathrm{C}-\mathrm{NMR}(125 \mathrm{~Hz}) ;{ }^{\mathrm{b}}$ tested in ${ }^{13} \mathrm{C}-\mathrm{NMR}(201 \mathrm{~Hz})$.

Table 2. ${ }^{1} \mathrm{H}-\mathrm{NMR}$ data of aglycone moieties for compounds $\mathbf{1 - 5}$ in $\mathrm{CD}_{3} \mathrm{OD}$.

\begin{tabular}{cccccc}
\hline \multirow{2}{*}{ Number } & \multicolumn{5}{c}{ Compounds $\left[\delta_{\mathbf{H}}\right.$ mult.(J in Hz) $]$} \\
\cline { 2 - 6 } & $\mathbf{1}^{\mathbf{a}}$ & $\mathbf{2}^{\mathbf{a}}$ & $\mathbf{3}^{\mathbf{b}}$ & $\mathbf{4}^{\mathbf{b}}$ & $\mathbf{5}^{\mathbf{a}}$ \\
\hline 1 & $3.37 \mathrm{~m}$ & $3.40 \mathrm{~m}$ & $3.44 \mathrm{~m}$ & $3.40 \mathrm{~m}$ & $3.40 \mathrm{~m}$ \\
2 & $1.70 \mathrm{~m}, 2.11$ & $1.72 \mathrm{~m}, 2.14$ & $1.78 \mathrm{~m}, 2.17$ & $1.78 \mathrm{~m}, 2.12$ & $1.80 \mathrm{~m}, 2.11$ \\
3 & $\mathrm{~m}$ & $\mathrm{~m}$ & $\mathrm{~m}$ & $\mathrm{~m}$ & $\mathrm{~m}$ \\
4 & $3.34 \mathrm{~m}$ & $3.38 \mathrm{~m}$ & $3.43 \mathrm{~m}$ & $3.38 \mathrm{~m}$ & $3.39 \mathrm{~m}$ \\
5 & $1.90 \mathrm{~m}$ & $2.22 \mathrm{~m}, 2.27$ & $2.22 \mathrm{~m}, 2.27$ & $2.24 \mathrm{~m}$ & $2.25 \mathrm{~m}$ \\
6 & - & $\mathrm{m}$ & $-\mathrm{m}$ & - & - \\
7 & $5.56 \mathrm{br} \mathrm{s}$ & $5.58 \mathrm{br} \mathrm{s}$ & $5.63 \mathrm{br} \mathrm{s}$ & $5.61 \mathrm{br} \mathrm{s}$ & $5.62 \mathrm{br} \mathrm{s}$ \\
8 & $1.30 \mathrm{~m}$ & $1.53 \mathrm{~m}, 1.97$ & $1.48 \mathrm{~m}, 2.87$ & $1.46 \mathrm{~m}, 2.87$ & $1.47 \mathrm{~m}, 2.84$ \\
9 & $1.56 \mathrm{~m}$ & $1.58 \mathrm{~m}$ & $2.26 \mathrm{~m}$ & $2.25 \mathrm{~m}$ & $2.24 \mathrm{~m}$ \\
10 & $1.25 \mathrm{~m}$ & $1.25 \mathrm{~m}$ & $1.47 \mathrm{~m}$ & $1.46 \mathrm{~m}$ & $1.47 \mathrm{~m}$ \\
11 & - & - & - & - & - \\
& $1.42 \mathrm{~m}, 2.54$ & $1.44 \mathrm{~m}, 2.55$ & $1.19 \mathrm{~m}, 2.94$ & $1.19 \mathrm{~m}, 2.98$ & $2.16 \mathrm{~m}$ \\
12 & $\mathrm{~m}$ & $\mathrm{~m}$ & $\mathrm{~m}$ & $\mathrm{~m}$ & \\
13 & $1.22 \mathrm{~m}, 1.65$ & $1.19 \mathrm{~m}, 1.72$ & $2.36 \mathrm{~m}, 2.60$ & $2.38 \mathrm{~m}, 2.59$ & $2.45 \mathrm{br} \mathrm{s}$ \\
14 & $\mathrm{~m}$ & $\mathrm{~m}$ & $\mathrm{~m}$ & $\mathrm{~m}$ & - \\
& $1.15 \mathrm{~m}$ & $1.78 \mathrm{~m}$ & - & - & - \\
\hline
\end{tabular}


Table 2. Cont.

\begin{tabular}{|c|c|c|c|c|c|}
\hline \multirow{2}{*}{ Number } & \multicolumn{5}{|c|}{ Compounds $\left[\delta_{\mathrm{H}}\right.$ mult. $(J$ in $\left.\mathrm{Hz})\right]$} \\
\hline & $1^{a}$ & $2^{a}$ & $3^{b}$ & $4^{b}$ & $5^{a}$ \\
\hline 15 & $\begin{array}{c}1.91 \mathrm{~m}, 1.97 \\
\mathrm{~m}\end{array}$ & $\begin{array}{c}1.45 \mathrm{~m}, 2.02 \\
\mathrm{~m}\end{array}$ & - & - & - \\
\hline 16 & $4.38 \mathrm{~m}$ & $4.53 \mathrm{q}(7.50)$ & $4.38 \mathrm{~d}(6.24)$ & $4.40 \mathrm{~d}(6.24)$ & $4.43 \mathrm{~m}$ \\
\hline 17 & $1.72 \mathrm{~m}$ & $1.78 \mathrm{~m}$ & $\begin{array}{c}2.34 \mathrm{dd} \\
(6.64,14.48)\end{array}$ & $\begin{array}{c}3.15 \mathrm{dd} \\
(6.56,7.84)\end{array}$ & $3.03 \mathrm{~m}$ \\
\hline 18 & $0.82 \mathrm{~s}$ & $0.94 \mathrm{~s}$ & - & - & - \\
\hline 19 & $1.12 \mathrm{~s}$ & $1.13 \mathrm{~s}$ & $1.09 \mathrm{~s}$ & $1.09 \mathrm{~s}$ & $1.10 \mathrm{~s}$ \\
\hline 20 & $1.90 \mathrm{~m}$ & $2.78 \mathrm{q}(7.00)$ & $3.14 \mathrm{~m}$ & $2.50 \mathrm{~m}$ & $2.08 \mathrm{~m}$ \\
\hline 21 & $0.96 \mathrm{~d}(6.90)$ & $\begin{array}{c}3.55 \mathrm{~m}, 3.69 \\
\mathrm{~m}\end{array}$ & $\begin{array}{c}3.74 \mathrm{~m}, 3.79 \\
\mathrm{~m}\end{array}$ & $\begin{array}{c}4.19 \mathrm{~m}, 4.33 \\
\mathrm{~m}\end{array}$ & $1.16 \mathrm{~d}(6.9)$ \\
\hline 22 & - & - & - & - & - \\
\hline 23 & $\begin{array}{c}1.44 \mathrm{~m}, 1.73 \\
\mathrm{~m}\end{array}$ & $3.52 \mathrm{~m}$ & $3.87 \mathrm{~m}$ & $3.33 \mathrm{~m}$ & $3.56 \mathrm{~m}$ \\
\hline 24 & $1.62 \mathrm{~m}$ & $3.76 \mathrm{~m}$ & $3.33 \mathrm{~m}$ & $3.34 \mathrm{~m}$ & $5.31 \mathrm{t}(2.9)$ \\
\hline 25 & $1.59 \mathrm{~m}$ & $1.91 \mathrm{~m}$ & $1.69 \mathrm{~m}$ & $1.67 \mathrm{~m}$ & $2.05 \mathrm{~m}$ \\
\hline 26 & $\begin{array}{c}3.30 \mathrm{~m}, 3.43 \\
\mathrm{~m}\end{array}$ & $\begin{array}{c}3.32 \mathrm{~m}, 3.54 \\
\mathrm{~m}\end{array}$ & $\begin{array}{c}3.49 \mathrm{~m}, 3.52 \\
\mathrm{~m}\end{array}$ & $\begin{array}{c}3.50 \mathrm{~m}, 3.53 \\
\mathrm{~m}\end{array}$ & $\begin{array}{c}3.35 \mathrm{~m}, 3.73 \\
\mathrm{~m}\end{array}$ \\
\hline 27 & $0.80 \mathrm{~d}(6.35)$ & $0.90 \mathrm{~d}(6.9)$ & $0.93 \mathrm{~d}(6.56)$ & $\begin{array}{l}0.94 \mathrm{~d}(6.56) \\
21-O \text {-acetyl }\end{array}$ & $\begin{array}{l}0.79 \mathrm{~d}(6.90) \\
24-O \text {-acetyl }\end{array}$ \\
\hline 1 & - & - & - & - & - \\
\hline 2 & - & - & - & $2.08 \mathrm{~s}$ & $2.16 \mathrm{~s}$ \\
\hline
\end{tabular}

a Tested in ${ }^{1} \mathrm{H}-\mathrm{NMR}(500 \mathrm{~Hz}){ }^{\mathrm{b}}$ tested in ${ }^{1} \mathrm{H}-\mathrm{NMR}(800 \mathrm{~Hz})$.

Table 3. ${ }^{13} \mathrm{C}-\mathrm{NMR}$ data of sugar portion of compound 1-5 in $\mathrm{CD}_{3} \mathrm{OD}$.

\begin{tabular}{|c|c|c|c|c|c|}
\hline \multirow{2}{*}{ Sugars } & \multicolumn{5}{|c|}{ Compounds $\left(\delta_{\mathrm{C}}\right)$} \\
\hline & $1^{\mathrm{a}}$ & $2^{a}$ & $3^{b}$ & $4^{b}$ & $5^{a}$ \\
\hline \multicolumn{6}{|l|}{$\operatorname{Ara}(p)$} \\
\hline 1 & 101.16 & 101.13 & 101.32 & 101.37 & 101.62 \\
\hline 2 & 74.58 & 74.56 & 71.22 & 71.11 & 74.40 \\
\hline 3 & 80.45 & 85.25 & 74.60 & 75.98 & 70.67 \\
\hline 4 & 85.29 & 70.52 & 76.16 & 75.62 & 85.51 \\
\hline 5 & 67.04 & 67.03 & 67.93 & 67.75 & 67.21 \\
\hline \multicolumn{6}{|l|}{ Xyl } \\
\hline 1 & 106.47 & 106.44 & & & 106.44 \\
\hline 2 & 74.91 & 74.89 & & & 74.88 \\
\hline 3 & 78.04 & 78.01 & & & 78.07 \\
\hline 4 & 70.54 & 70.78 & & & 71.19 \\
\hline 5 & 67.04 & 67.03 & & & 67.02 \\
\hline \multicolumn{6}{|l|}{ Rha } \\
\hline 1 & 101.60 & 101.58 & 98.43 & 98.99 & 101.78 \\
\hline 2 & 71.99 & 71.98 & 73.46 & 73.87 & 72.39 \\
\hline 3 & 74.58 & 80.45 & 75.74 & 77.91 & 72.17 \\
\hline 4 & 73.05 & 73.04 & 74.65 & 73.46 & 74.29 \\
\hline 5 & 69.84 & 69.86 & 67.34 & 69.81 & 69.83 \\
\hline 6 & 18.72 & 18.71 & 18.25 & 18.58 & 18.65 \\
\hline \multicolumn{6}{|l|}{ 2-acetyl } \\
\hline 1 & - & - & 171.96 & 172.11 & \\
\hline 2 & - & - & 21.02 & 21.09 & \\
\hline \multicolumn{6}{|l|}{4 -acetyl } \\
\hline 1 & - & - & 172.29 & - & \\
\hline 2 & - & - & 21.15 & - & \\
\hline \multicolumn{6}{|l|}{ Api } \\
\hline 1 & 112.17 & 112.15 & 112.54 & 112.37 & \\
\hline 2 & 78.23 & 78.28 & 78.49 & 78.34 & \\
\hline 3 & 80.49 & 80.48 & 80.68 & 80.71 & \\
\hline 4 & 75.18 & 75.17 & 75.41 & 75.35 & \\
\hline 5 & 65.56 & 65.58 & 65.56 & 65.77 & \\
\hline
\end{tabular}


Table 4. ${ }^{1} \mathrm{H}-\mathrm{NMR}$ data of the sugar portion of compounds $\mathbf{1}-\mathbf{5}$ in $\mathrm{CD}_{3} \mathrm{OD}$.

\begin{tabular}{|c|c|c|c|c|c|}
\hline \multirow{2}{*}{ Sugars } & \multicolumn{5}{|c|}{ Compounds $\left[\delta_{\mathrm{H}}\right.$ mult. $(J$ in $\left.\mathrm{Hz})\right]$} \\
\hline & $1^{a}$ & $2^{a}$ & $3^{b}$ & $4^{b}$ & $5^{a}$ \\
\hline \multicolumn{6}{|l|}{$\operatorname{Ara}(p)$} \\
\hline 1 & $4.34 \mathrm{~d}(7.35)$ & $4.34 \mathrm{~d}(7.35)$ & $4.52 \mathrm{~d}(7.60)$ & $4.30 \mathrm{~d}(7.44)$ & $4.31 \mathrm{~d}(7.55)$ \\
\hline 2 & $3.83 \mathrm{~m}$ & $3.83 \mathrm{~m}$ & $3.74 \mathrm{~m}$ & $3.73 \mathrm{~m}$ & $3.86 \mathrm{~m}$ \\
\hline 3 & $3.70 \mathrm{~m}$ & $3.76 \mathrm{~m}$ & $3.76 \mathrm{~m}$ & $3.69 \mathrm{~m}$ & $4.00 \mathrm{~m}$ \\
\hline 4 & $3.76 \mathrm{~m}$ & $3.99 \mathrm{~m}$ & $3.72 \mathrm{~m}$ & $3.73 \mathrm{~m}$ & $3.76 \mathrm{~m}$ \\
\hline 5 & $3.22 \mathrm{~m}, 3.49 \mathrm{~m}$ & $3.51 \mathrm{~m}, 3.86 \mathrm{~m}$ & $3.52 \mathrm{~m}, 3.83 \mathrm{~m}$ & $3.52 \mathrm{~m}, 3.83 \mathrm{~m}$ & $3.54 \mathrm{~m}, 3.85 \mathrm{~m}$ \\
\hline \multicolumn{6}{|l|}{ Xyl } \\
\hline 1 & $4.41 \mathrm{br} \mathrm{d}(7.10)$ & $4.43 \mathrm{~d}(7.15)$ & & & $4.44 \mathrm{~d}(7.16)$ \\
\hline 2 & $3.27 \mathrm{~m}$ & $3.30 \mathrm{~m}$ & & & $3.31 \mathrm{~m}$ \\
\hline 3 & $3.30 \mathrm{~m}$ & $3.33 \mathrm{~m}$ & & & $3.34 \mathrm{~m}$ \\
\hline 4 & $3.96 \mathrm{~m}$ & $3.71 \mathrm{~m}$ & & & $3.53 \mathrm{~m}$ \\
\hline 5 & $3.49 \mathrm{~m}, 3.84 \mathrm{~m}$ & $3.51 \mathrm{~m}, 3.86 \mathrm{~m}$ & & & $3.24 \mathrm{~m}, 3.88 \mathrm{~m}$ \\
\hline \multicolumn{6}{|l|}{ Rha } \\
\hline 1 & $5.31 \mathrm{br} \mathrm{s}$ & 5.33 br s & 5.37 br s & $5.28 \mathrm{~m}$ & 5.35 br s \\
\hline 2 & $4.06 \mathrm{~m}$ & $4.09 \mathrm{~m}$ & $\begin{array}{c}5.29 \mathrm{dd}(1.76, \\
3.36)\end{array}$ & $5.28 \mathrm{~m}$ & $3.93 \mathrm{br} \mathrm{s}$ \\
\hline 3 & $3.83 \mathrm{~m}$ & $3.71 \mathrm{~m}$ & $4.08 \mathrm{~m}$ & $3.86 \mathrm{~m}$ & $3.69 \mathrm{~m}$ \\
\hline 4 & $3.49 \mathrm{~m}$ & $3.52 \mathrm{~m}$ & $4.95 \mathrm{t}(9.92)$ & $3.46 \mathrm{~m}$ & $3.41 \mathrm{~m}$ \\
\hline 5 & $4.11 \mathrm{~m}$ & $\begin{array}{c}4.14 \mathrm{dd}(6.20 \\
9.55)\end{array}$ & $4.46 \mathrm{~m}$ & $4.21 \mathrm{~m}$ & $4.14 \mathrm{~m}$ \\
\hline 6 & $1.25 \mathrm{~d}(6.15)$ & $1.27 \mathrm{~d}(6.15)$ & $1.14 \mathrm{~d}(6.24)$ & $1.26 \mathrm{~d}(6.16)$ & $1.26 \mathrm{~d}(6.15)$ \\
\hline \multicolumn{6}{|l|}{ 2-acetyl } \\
\hline 1 & - & - & - & - & - \\
\hline 2 & - & - & $2.02 \mathrm{~s}$ & $2.08 \mathrm{~s}$ & - \\
\hline \multicolumn{6}{|l|}{ 4-acetyl } \\
\hline 1 & - & - & - & - & - \\
\hline 2 & - & - & $2.12 \mathrm{~s}$ & - & - \\
\hline \multicolumn{6}{|l|}{ Api } \\
\hline 1 & $5.19 \mathrm{~d}(2.90)$ & $5.21 \mathrm{~d}(3.60)$ & $5.04 \mathrm{~d}(2.24)$ & $5.18 \mathrm{~d}(2.0)$ & \\
\hline 2 & $4.02 \mathrm{~m}$ & $4.02 \mathrm{~m}$ & $3.81 \mathrm{~m}$ & $3.93 \mathrm{~m}$ & \\
\hline 3 & - & - & - & - & \\
\hline 4 & $3.76 \mathrm{~m}, 4.07 \mathrm{~m}$ & $3.79 \mathrm{~m}, 4.09 \mathrm{~m}$ & $3.72 \mathrm{~m}, 3.93 \mathrm{~m}$ & $3.72 \mathrm{~m}, 3.92 \mathrm{~m}$ & \\
\hline 5 & $3.62 \mathrm{~m}, 3.86 \mathrm{~m}$ & $3.64 \mathrm{~m}$ & $3.56 \mathrm{~m}, 3.65 \mathrm{~m}$ & $3.55 \mathrm{~m}$ & \\
\hline
\end{tabular}

a Tested in ${ }^{1} \mathrm{H}-\mathrm{NMR}(500 \mathrm{~Hz}) ;{ }^{\mathrm{b}}$ tested in ${ }^{1} \mathrm{H}-\mathrm{NMR}(800 \mathrm{~Hz})$.
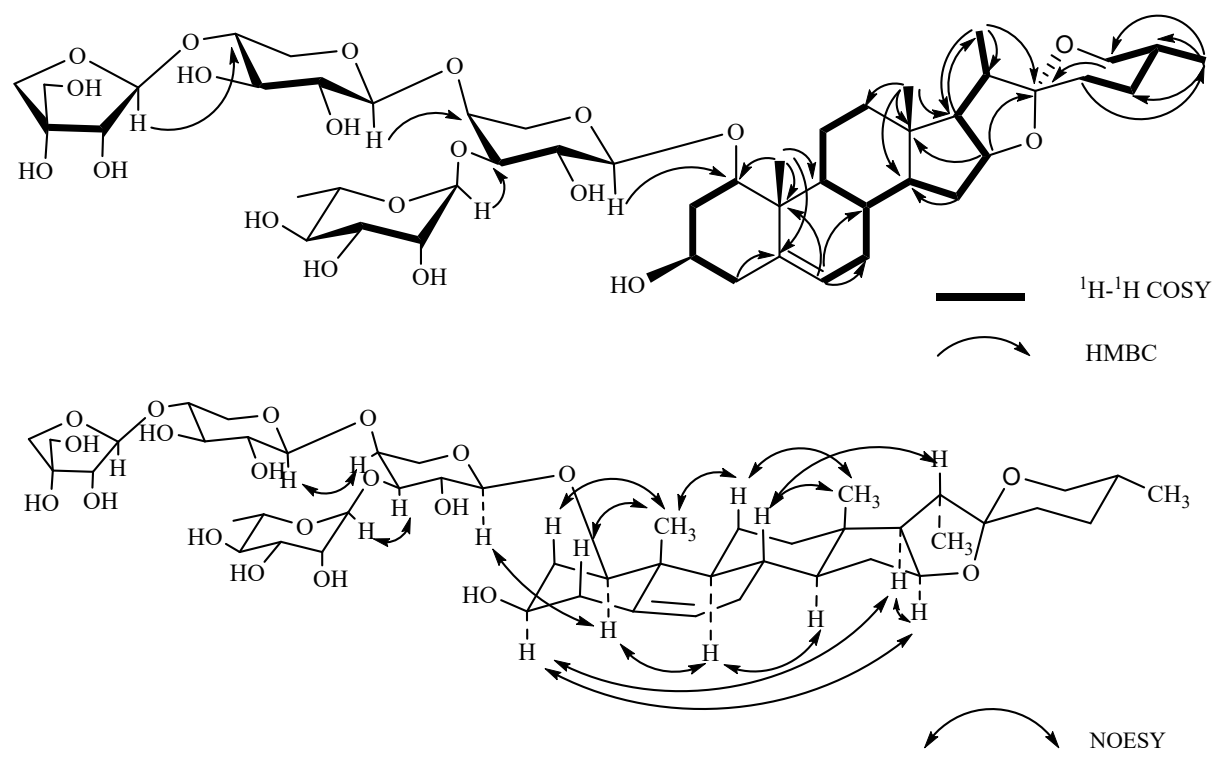

Figure 2. Key ${ }^{1} \mathrm{H}^{-1} \mathrm{H}$ COSY, HMBC, and NOESY correlations of compound $\mathbf{1 .}$

Compound 2, named Pamaiosides B, a white amorphous solid, was positive to Liebermann Burchard and Molisch chemical reactions. The pseudomolecular ion peak was measured in the HR-ESI-MS spectrum at $m / z 1043.4677[\mathrm{M}+\mathrm{Na}]^{+}$(calculated for $\mathrm{C}_{48} \mathrm{H}_{76} \mathrm{O}_{23} \mathrm{Na}$, 1043.4675), corresponding to the molecular formula $\mathrm{C}_{48} \mathrm{H}_{76} \mathrm{O}_{23}$. Com- 
pared to 1, one angular methyl at $\delta_{\mathrm{C}} 15.07$ (C-21) and two methylenes at $\delta_{\mathrm{C}} 32.59$ (C-23) and 30.04 (C-24) were absent, and the chemical shifts were all markedly up-field at $\delta_{\mathrm{C}} 62.95\left(\mathrm{C}-21, \Delta \delta_{\mathrm{C}}+47.88 \mathrm{ppm}\right), \delta_{\mathrm{C}} 71.20\left(\mathrm{C}-23, \Delta \delta_{\mathrm{C}}+38.61 \mathrm{ppm}\right)$, and $\delta_{\mathrm{C}} 74.01(\mathrm{C}-24$, $\left.\Delta \delta_{\mathrm{C}}+43.97 \mathrm{ppm}\right)$, respectively, which indicates that a hydroxyl group substituted at the primary carbon atom (Tables 1-4). In the HMBC spectra, the cross-peaks between $\delta_{\mathrm{H}}$ $2.78(\mathrm{H}-20)$ and $\delta_{\mathrm{C}} 62.95(\mathrm{C}-21)$ and between $\delta_{\mathrm{Ha}} 3.55, \delta_{\mathrm{Hb}} 3.69(\mathrm{H}-21)$ and $\delta_{\mathrm{C}} 46.04(\mathrm{C}-20) /$ $\delta_{\mathrm{C}} 112.72$ (C-22) authenticated the hydroxyl substituted at C-21, and it was further confirmed by the correlations for $\delta_{\mathrm{Ha}} 3.55, \delta_{\mathrm{Hb}} 3.69(\mathrm{H}-21)$ to $\delta_{\mathrm{H}} 2.78(\mathrm{H}-20)$ in the ${ }^{1} \mathrm{H}-{ }^{1} \mathrm{H}$ COSY spectrum (Figure 3). Meanwhile, the correlations for $\delta_{\mathrm{H}} 1.91(\mathrm{H}-25)$ to $\delta_{\mathrm{H}} 3.76(\mathrm{H}-24)$ and $\delta_{\mathrm{H}} 3.76(\mathrm{H}-24)$ to $\delta_{\mathrm{H}} 3.52(\mathrm{H}-23)$ in the ${ }^{1} \mathrm{H}-{ }^{1} \mathrm{H}$ COSY spectrum and the signal of $\delta_{\mathrm{H}} 0.90(\mathrm{H}-27)$ to $\delta_{\mathrm{C}} 74.01(\mathrm{C}-24)$ derived from the HMBC spectrum illustrated that the hydroxyl displaced at C-23 and C-24. In the NOESY spectra, the configurations of C-1, C-3, $\mathrm{C}-23$, and $\mathrm{C}-24$ were successively evidenced as $\beta, \beta, \alpha$, and $\beta$ orientations derived from correlations for $\mathrm{H}-1\left(\delta_{\mathrm{H}} 3.40\right)$ to $\mathrm{H}-9\left(\delta_{\mathrm{H}} 1.25\right), \mathrm{H}-3\left(\delta_{\mathrm{H}} 3.38\right)$ to $\mathrm{H}-9\left(\delta_{\mathrm{H}} 1.25\right), \mathrm{H}-20\left(\delta_{\mathrm{H}} 2.78\right)$ to $\mathrm{H}-23\left(\delta_{\mathrm{H}} 3.52\right)$, and $\mathrm{H}-24\left(\delta_{\mathrm{H}} 3.76\right)$ to $\mathrm{H}-27\left(\delta_{\mathrm{H}} 0.90\right)$, respectively. Using the same method as for $1, C-20, C-22$, and C-25 were determined as $R$ configuration. By summarizing the data and comparing it to the literature [22], the aglycone of compound 2 was established as $(20 R, 22 R, 25 R)$-spirost-5-en- $1 \beta, 3 \beta, 21,23 \alpha, 24 \beta$-pentol.

Acid hydrolysis, derivatization, and GC analysis revealed that compound 2 possessed the same monosaccharide residues as $\mathbf{1}$, but different linkages emerged between the sugars. In the HMBC spectra, the sugar sequencing linkages were testified by the correlations between Api H-1 $\left(\delta_{\mathrm{H}} 5.21\right)$ and Rha C-3 $\left(\delta_{\mathrm{C}} 80.45\right)$, Rha H-1 $\left(\delta_{\mathrm{H}} 5.33\right)$ and $\mathrm{Xyl} \mathrm{C-2}\left(\delta_{\mathrm{C}} 74.89\right)$, Xyl H-1 $\left(\delta_{\mathrm{H}} 4.43\right)$ and Ara C-3 $\left(\delta_{\mathrm{C}} 85.25\right)$, and Ara H-1 $\left(\delta_{\mathrm{H}} 4.34\right)$ and $\mathrm{C}-1\left(\delta_{\mathrm{C}} 84.80\right)$. Thus, compound 2 was elucidated as $(20 R, 22 R, 25 R)$-spirost-5-en- $1 \beta, 3 \beta, 21,23 \alpha, 24 \beta$-pentol-1-O- $\beta$-D-apiofuranosyl$(1 \rightarrow 3)$ - $\alpha$-L-rhamnopyranosyl-( $1 \rightarrow 2)-\beta$-D-xylopyranosyl-( $1 \rightarrow 3)-a$-L-arabinopyranoside.
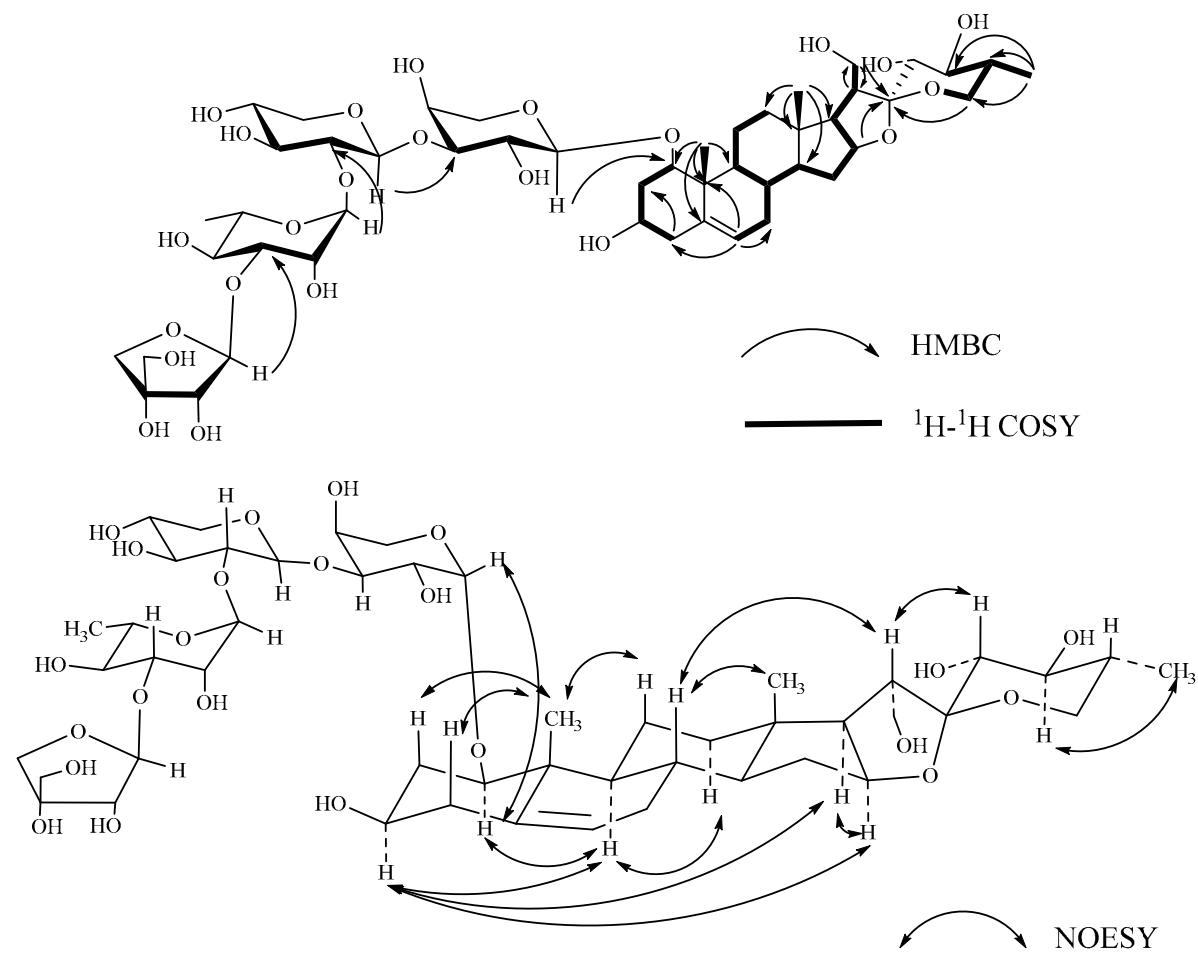

Figure 3. Key ${ }^{1} \mathrm{H}-{ }^{1} \mathrm{H}$ COSY, HMBC, and NOESY correlations of compound 2.

Compound 3, named Pamaiosides C, a white amorphous solid, was positive to Liebermann Burchard and Molisch chemical reactions. The pseudomolecular ion peak was measured in the HR-ESI-MS spectrum at $m / z 993.3932[\mathrm{M}+\mathrm{Na}]^{+}$(calculated for $\mathrm{C}_{46} \mathrm{H}_{66} \mathrm{O}_{22} \mathrm{Na}$, 993.3943), corresponding to the molecular formula $\mathrm{C}_{46} \mathrm{H}_{66} \mathrm{O}_{22}$. Compared to 2, one angular methyl $\delta_{\mathrm{C}} 17.30(\mathrm{C}-18)$ was missing and two quaternary carbons, $\delta_{\mathrm{C}} 179.23(\mathrm{C}-13)$ and 
139.51 (C-14), and one ketone $\delta_{\mathrm{C}} 207.09$ (C-15) signal were detected (Tables 1-4). In the $\mathrm{HMBC}$ spectra, the cross-peaks between $\delta_{\mathrm{Ha}} 1.19, \delta_{\mathrm{Hb}} 2.94(\mathrm{H}-11) / \delta_{\mathrm{Ha}} 2.36, \delta_{\mathrm{Hb}} 2.60(\mathrm{H}-12) /$ $\delta_{\mathrm{H}} 2.34(\mathrm{H}-17) / \delta_{\mathrm{H}} 4.38(\mathrm{H}-16)$ and $\delta_{\mathrm{C}} 179.23(\mathrm{C}-13)$, between $\delta_{\mathrm{H}} 2.26(\mathrm{H}-8) / \delta_{\mathrm{Ha}} 1.48, \delta_{\mathrm{Hb}}$ $2.87(\mathrm{H}-7)$, and $\delta_{\mathrm{C}} 139.51(\mathrm{C}-14)$ allowed to deduce that one double bond was located at $\mathrm{C}-13 / \mathrm{C}-14$. Moreover, the location of $\delta_{\mathrm{C}} 207.09$ (C-15) was affirmed by correlation of $\delta_{\mathrm{H}}$ $2.34(\mathrm{H}-17)$ to $\delta_{\mathrm{C}} 207.09$ (C-15) (Figure 4 ). As a result, the aglycone of 3 was determined as 15-oxo-18-nor-(20R,22R,25R)-spirost-5,13-diene-1 $\beta, 3 \beta, 21,23 \alpha, 24 \beta$-pentol [23].

The monosaccharide residues were identified as L-Ara, L-Rha, and D-Api in a ratio of 1:1:1 by acid hydrolysis, derivatization, and GC analysis. In addition, two ketomethyls at $\delta_{\mathrm{C}} 21.15\left(3 \mathrm{H}, \mathrm{s}, \delta_{\mathrm{H}} 2.12\right)$ and $21.02\left(3 \mathrm{H}, s, \delta_{\mathrm{H}} 2.02\right)$ and two carbonyl at $\delta_{\mathrm{C}} 172.29$ and 171.96 carbons signals were observed, which infers that two acetyl groups existed in the sugar chain. In the HMBC spectrum, one proton of keto-methyl at $\delta_{\mathrm{H}} 2.12$ was correlated with one carbonyl carbon signal at $\delta_{\mathrm{C}} 172.29$ and $\delta_{\mathrm{C}} 74.65$ (C-4, Rha); moreover, H-4 of Rha $\left(\delta_{\mathrm{H}} 4.95\right)$ was correlated with $\delta_{\mathrm{C}} 172.29$, suggesting that one acetyl was connected at C-4 of Rha. In the same way, another acetyl was substituted at C-2 of Rha, elaborated by the cross-peaks between $\delta_{\mathrm{H}} 2.02$ and $\delta_{\mathrm{C}} 171.96 / \delta_{\mathrm{C}} 73.46(\mathrm{C}-2, \mathrm{Rha})$ and between $\delta_{\mathrm{H}} 5.29\left(\mathrm{H}-2\right.$, Rha) and $\delta_{\mathrm{C}}$ 171.96. Compared to 2, it was further confirmed by the up-field shifts of $\delta_{\mathrm{H}} 5.29\left(\mathrm{H}-2\right.$ of Rha, $\left.\Delta \delta_{\mathrm{C}}+1.2 \mathrm{ppm}\right)$ and $\delta_{\mathrm{H}} 4.95(\mathrm{H}-4$ of Rha, $\left.\Delta \delta_{\mathrm{C}}+1.43 \mathrm{ppm}\right)$. The $\beta$ configuration of $\mathrm{D}$-apiose affirmed the chemical shifts of $\delta_{\mathrm{C}} 112.54(\mathrm{C}-1), 78.49(\mathrm{C}-2), 80.68(\mathrm{C}-3), 75.41(\mathrm{C}-4)$, and 65.56 (C-5) [20]. The $a$ configuration of L-rhamnopyranosyl was confirmed by the chemical shifts of Rha C-5 at $\delta_{\mathrm{C}} 67.34$ [21]. The anomeric proton coupling constants of L-arabopyranose $(J=7.6 \mathrm{~Hz}>7.0 \mathrm{~Hz}) \mathrm{sug}$ gests that the configuration was an $\alpha$ orientation [19]. Thus, compound 3 was determined as 15-oxo-18-nor-(20R,22R,25R)-spirost-5,13-diene- $1 \beta, 3 \beta, 21,23 \alpha, 24 \beta$-pentol-1-O- $\beta$-Dapiofuranosyl-( $(\rightarrow 3)$-2,4-diacetyl- $\alpha$-L-rhamnopyranosyl- $(1 \rightarrow 3)$ - $a$-L-arabinopyranoside.
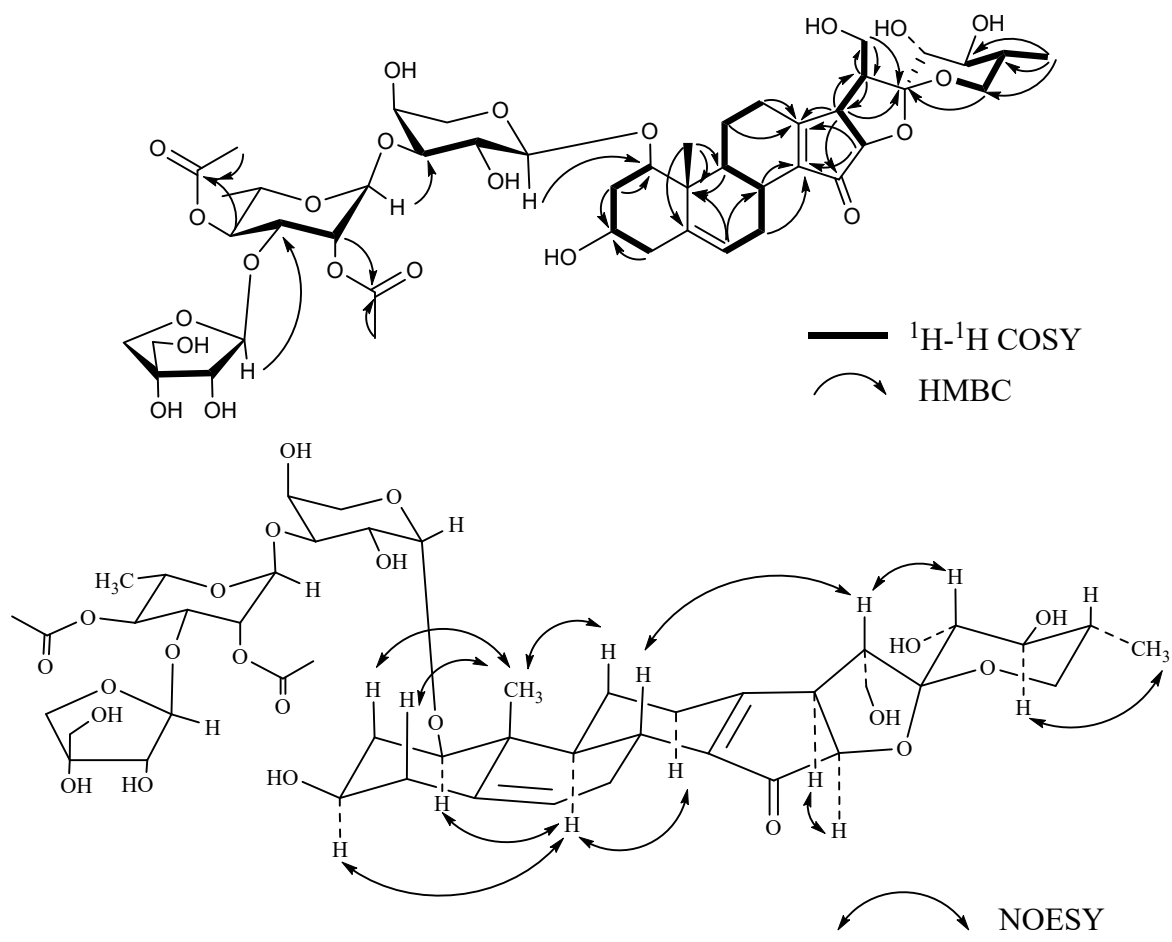

Figure 4. Key ${ }^{1} \mathrm{H}-{ }^{1} \mathrm{H}$ COSY, HMBC, and NOESY correlations of compound 3.

Compound 4, named Pamaiosides D, a white amorphous solid, was positive to Liebermann Burchard and Molisch chemical reactions. The pseudomolecular ion peak was measured in the HR-ESI-MS spectrum at $m / z 993.3969[\mathrm{M}+\mathrm{Na}]^{+}$(calculated for $\mathrm{C}_{46} \mathrm{H}_{66} \mathrm{O}_{22} \mathrm{Na}$, 993.3943), corresponding to the molecular formula $\mathrm{C}_{46} \mathrm{H}_{66} \mathrm{O}_{22}$. Compared with 3, only two distinctions, the position of one acetyl group and the sugar linkages, were detected 
(Tables 1-4). The acetyl group replaced at $\mathrm{C}-21$, which was evidenced by the altered proton chemical shifts at $\delta_{\mathrm{H}} 3.46\left(\mathrm{H}-4\right.$ of Rha, $\left.\Delta \delta_{\mathrm{C}}-1.49 \mathrm{ppm}\right), \delta_{\mathrm{Ha}} 4.19$ (Ha-21, $\left.\Delta \delta_{\mathrm{C}}+0.45 \mathrm{ppm}\right)$, and $\delta_{\mathrm{Hb}} 4.33\left(\mathrm{Hb}-21, \Delta \delta_{\mathrm{C}}+0.54 \mathrm{ppm}\right)$. It was further acknowledged by the cross-peaks between $\delta_{\mathrm{H}} 2.08\left(3 \mathrm{H}, s, \mathrm{CH}_{3} \mathrm{CO}-\right) / \delta_{\mathrm{Ha}} 4.19, \delta_{\mathrm{Hb}} 4.33(\mathrm{H}-21)$, and $\delta_{\mathrm{C}} 172.90\left(\mathrm{CH}_{3} \underline{\mathrm{CO}}-\right)$ in the HMBC spectrum (Figure 5). In addition, the correlations for Api H-1 ( $\left.\delta_{\mathrm{H}} 5.18\right)$ to Rha C-3 $\left(\delta_{\mathrm{C}} 77.91\right)$, Rha H-1 $\left(\delta_{\mathrm{H}} 5.28\right)$ to Ara C-4 $\left(\delta_{\mathrm{C}} 75.62\right)$, and Ara H-1 $\left(\delta_{\mathrm{H}} 4.30\right)$ to $\mathrm{C}-1\left(\delta_{\mathrm{C}} 85.33\right)$ in the HMBC spectra clarified the linkages. Thus, compound 4 was characterized as 15-oxo-18-nor-(20R,22R,25R)-spirost-5,13-diene-21-O-acetyl-1 $\beta, 3 \beta, 21,23 \alpha, 24 \beta$-pentol-1$O$ - $\beta$-D-apiofuranosyl-( $1 \rightarrow 3)$-2-acetyl- $\alpha$-L-rhamnopyranosyl-( $1 \rightarrow 4)-a$-L-arabinopyranoside.
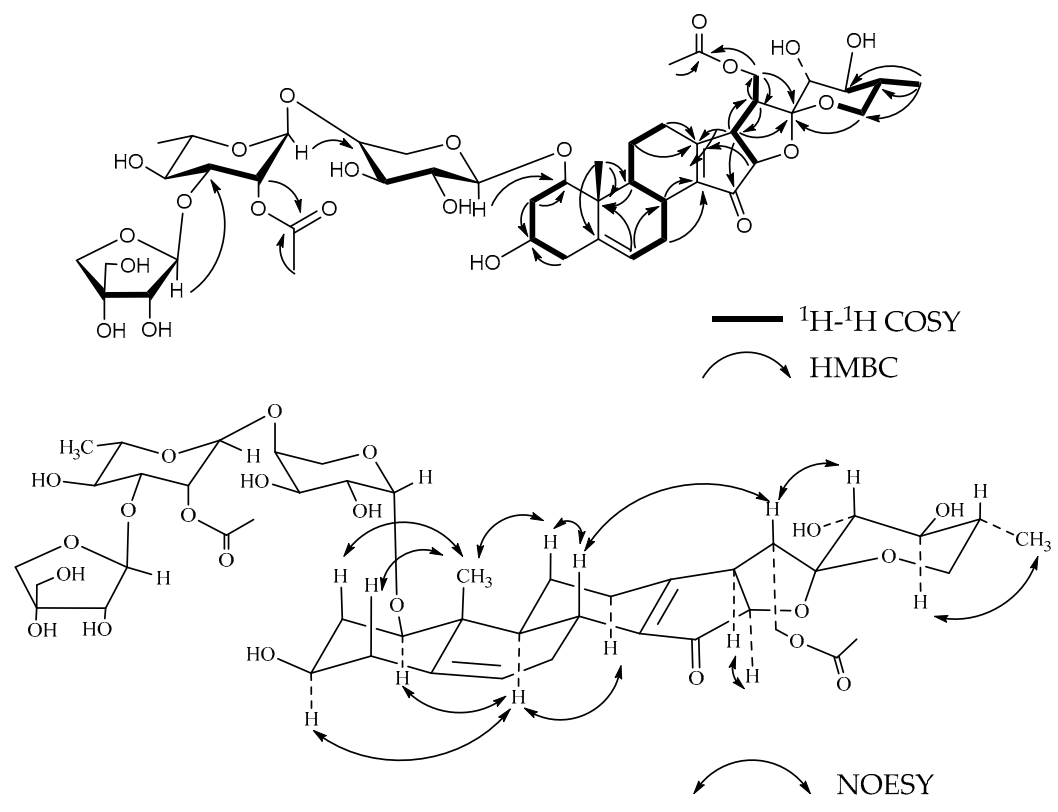

Figure 5. Key ${ }^{1} \mathrm{H}^{-1} \mathrm{H}$ COSY, HMBC, and NOESY correlations of compound 4.

Compound 5, named Pamaiosides E, a white amorphous solid, was positive to Liebermann Burchard and Molisch chemical reactions. The pseudomolecular ion peak was measured in the HR-ESI-MS spectrum at $\mathrm{m} / \mathrm{z} 935.3892[\mathrm{M}+\mathrm{Na}]^{+}$(calculated for $\mathrm{C}_{44} \mathrm{H}_{64} \mathrm{O}_{20} \mathrm{Na}$, 935.3889), corresponding to the molecular formula $\mathrm{C}_{44} \mathrm{H}_{64} \mathrm{O}_{20}$. Compared to 3 , the proton signals at $\mathrm{H}-21$ were replaced by one angular methyl, $\delta_{\mathrm{H}} 1.16(3 \mathrm{H}, d)$, in an aglycone moiety. Moreover, one keto-methyl at $21.27\left(3 \mathrm{H}, s, \delta_{\mathrm{H}} 2.16\right)$ and one carbonyl at $\delta_{\mathrm{C}} 173.78$ signals were observed in the ${ }^{13} \mathrm{C}-\mathrm{NMR}$ spectra (Tables $\left.1-4\right)$. By analyzing the HMBC spectrum, the cross-peaks between $\delta_{\mathrm{H}} 2.16\left(\mathrm{CH}_{3} \mathrm{CO}-\right) / \delta_{\mathrm{H}} 5.31(\mathrm{H}-24)$ and $\delta_{\mathrm{C}} 173.78\left(\mathrm{CH}_{3} \mathrm{CO}\right)$ conjectured that one acetyl was substituted at $\mathrm{C}-24$, and the up-field chemical shifts at $\mathrm{H}-24(\Delta \mathrm{ppm}+1.98)$ proved the hypothesis (Figure 6). According to the methodology, C-1, C-3, C-23, and C-24 possessed the same configuration as compound 3, and the configurations of C-20, C-22, and C-25 were decided as $S, S$, and $R$, respectively. Therefore, the aglycone of 5 was determined as 15 -oxo-18-nor-(20S,22S,25R)-spirost-5,13diene-24-acetyl-1 $\beta, 3 \beta, 23 \alpha, 24 \beta$-tetrol.

Acid hydrolysis and GC analysis of 5 exhibited L-Ara, L-Rha, and D-Xyl residues in a ratio of 1:1:1. The configuration of each monosaccharide was deduced by the same approach employed in compound $\mathbf{1}$, which was $\alpha$-L-Ara, $\alpha$-L-Rha, and $\beta$-D-Xyl, respectively. The sequence was derived from the correlations from Xyl H-1 $\left(\delta_{\mathrm{H}} 4.44\right)$ to Ara C-4 $\left(\delta_{\mathrm{C}} 85.51\right)$, Rha $\mathrm{H}-1\left(\delta_{\mathrm{H}} 5.35\right)$ to Ara C-2 $\left(\delta_{\mathrm{C}} 74.40\right)$, and Ara H-1 $\left(\delta_{\mathrm{H}} 4.31\right)$ to C-1 $\left(\delta_{\mathrm{C}} 85.57\right)$. Thus, compound 5 was identified as 15 -oxo-18-nor-(20S,22S,25R)-spirost-5,13-diene-24-acetyl-1 $\beta, 3 \beta, 23 \alpha, 24 \beta$ tetrol-1-O- $\beta$-D-xylopyranosyl-( $1 \rightarrow 4)$-[ $\alpha$-L-rhamnopyranosyl-( $(1 \rightarrow 2)]-a$-L-arabinopyranoside. 


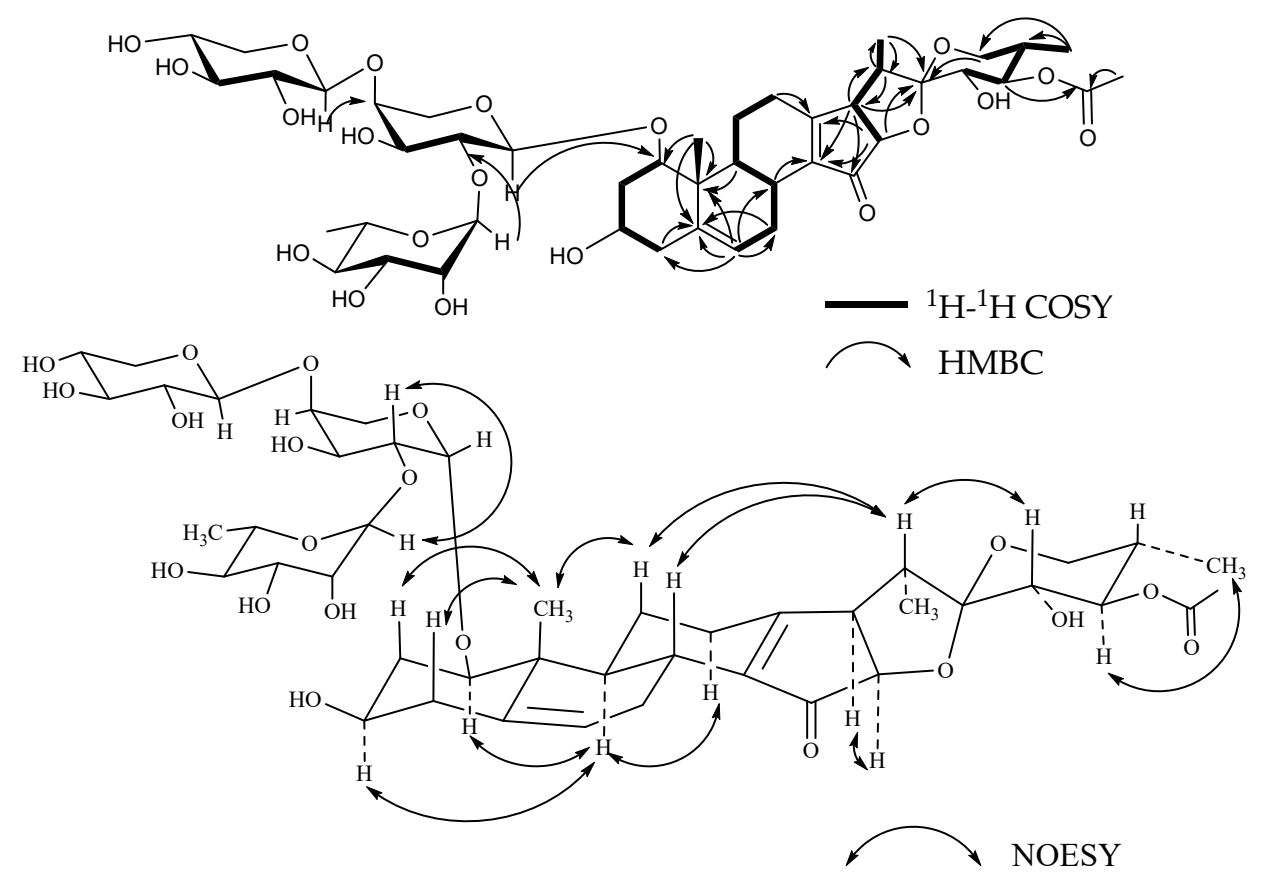

Figure 6. Key ${ }^{1} \mathrm{H}^{-1} \mathrm{H}$ COSY, HMBC, and NOESY correlations of compound 5.

The five known steroidal saponins, 6-10, were defined as $25(R)$-spirost-5-en- $1 \beta, 3 \beta, 21,23 \alpha, 24 \beta$ pentol-1-O- $\beta$-D-apiofuranosyl-( $1 \rightarrow 3)-\alpha$-L-rhamnopyranosyl- $(1 \rightarrow 2)-[\beta$-D-xylopyranosyl- $(1 \rightarrow 4)]-a$-Larabinopyranoside (6) [21]; 15-oxo-18-nor-25(R)-spirost-5,13-diene-1 $\beta, 3 \beta, 21,23 \alpha, 24 \beta$-pentol-1-O- $\beta$-Dapiofuranosyl-( $(1 \rightarrow 3)-\alpha$-L-rhamnopyranosyl-( $1 \rightarrow 2)$-[ $\beta$-D-xylopyranosyl-( $1 \rightarrow 3)]-a$-L-arabinopyranosi de (7) [24]; 15-oxo-18-nor-25(R)-spirost-5,13-diene-24-acetyl-1 $\beta, 3 \beta, 23 \alpha, 24 \beta$-tetrol-1-O- $\beta$-D-apiofuranos yl- $(1 \rightarrow 3)-\alpha$-L-rhamnopyranosyl-( $1 \rightarrow 2)$-[ $\beta$-D-xylopyranosyl- $(1 \rightarrow 3)]$ - $a$-L-arabinopyranoside $(8)$ [25]; 15-oxo-18-nor-25(R)-spirost-5,13-diene-1 $\beta, 3 \beta, 21,23 \alpha, 24 \beta$-pentol-1-O- $\beta$-D-apiofuranosyl-( $1 \rightarrow 3)-\alpha$-L-rh amnopyranosyl-(1 $\rightarrow 2)$-[ $\beta$-D-xylopyranosyl-(1 $\rightarrow 4)$ ]- $a$-L-arabinopyranoside $(9)$ [26]; 25(R)-spirost-5en-1 $\beta, 3 \beta, 21,23 \alpha, 24 \beta$-pentol-1-O- $\beta$-D- $\alpha$-L-rhamnopyranosyl-(1 $\rightarrow 2)$-[ $\beta$-D-xylopyranosyl-(1 $\rightarrow 3)]$ - $a$-L-ar abinopyranoside (10) [27] (Tables 5 and 6) by comparison of the physical and spectroscopic data available in the literature.

Table 5. ${ }^{13} \mathrm{C}-\mathrm{NMR}$ data of the aglycone moieties of compounds $\mathbf{6}-\mathbf{1 0}$.

\begin{tabular}{cccccc}
\hline \multirow{2}{*}{ Number } & \multicolumn{5}{c}{ Compounds } \\
\cline { 2 - 5 } & $\mathbf{6}^{\mathbf{b}}$ & $\mathbf{7}^{\mathbf{a}}$ & $\mathbf{8}^{\mathbf{a}}$ & $\mathbf{9}^{\mathbf{a}}$ & $\mathbf{1 0}^{\mathbf{a}}$ \\
\hline 1 & 84.74 & 85.37 & 85.42 & 85.37 & 84.98 \\
2 & 38.18 & 37.56 & 37.62 & 37.56 & 37.45 \\
3 & 68.87 & 69.35 & 69.40 & 69.38 & 69.28 \\
4 & 44.38 & 42.94 & 42.96 & 42.95 & 43.59 \\
5 & 140.08 & 139.73 & 139.72 & 139.72 & 139.80 \\
6 & 125.40 & 126.16 & 126.16 & 126.22 & 126.10 \\
7 & 32.58 & 30.26 & 30.29 & 30.31 & 32.86 \\
8 & 33.75 & 32.82 & 32.82 & 32.88 & 34.25 \\
9 & 50.94 & 48.77 & 48.74 & 48.80 & 51.56 \\
10 & 43.51 & 43.33 & 43.34 & 43.36 & 43.59 \\
11 & 24.60 & 26.26 & 26.38 & 26.25 & 24.80 \\
12 & 40.99 & 29.28 & 29.29 & 29.21 & 41.24 \\
13 & 41.46 & 179.29 & 179.38 & 179.31 & 41.79 \\
14 & 57.61 & 139.89 & 139.60 & 139.85 & 58.26 \\
15 & 33.05 & 207.37 & 208.15 & 207.08 & 33.14 \\
16 & 84.11 & 83.15 & 82.96 & & 84.36 \\
\hline
\end{tabular}


Table 5. Cont.

\begin{tabular}{cccccc}
\hline \multirow{2}{*}{ Number } & \multicolumn{5}{c}{ Compounds } \\
\cline { 2 - 6 } & $\mathbf{6}^{\mathbf{b}}$ & $\mathbf{7}^{\mathbf{a}}$ & $\mathbf{8}^{\mathbf{a}}$ & $\mathbf{9}^{\mathbf{a}}$ & $\mathbf{1 0}^{\mathbf{a}}$ \\
\hline 17 & 58.37 & 48.77 & 52.40 & 49.37 & 58.61 \\
18 & 17.58 & - & - & - & 17.32 \\
19 & 15.69 & 14.41 & 14.40 & 14.41 & 15.50 \\
20 & 46.25 & 50.13 & 43.69 & 49.49 & 46.03 \\
21 & 62.99 & 62.03 & 14.06 & 61.98 & 62.94 \\
22 & 113.19 & 114.18 & 113.50 & 114.84 & 112.71 \\
23 & 70.99 & 70.64 & 67.56 & 74.51 & 71.19 \\
24 & 73.65 & 73.32 & 73.89 & 76.25 & 73.96 \\
25 & 36.61 & 36.38 & 35.42 & 39.30 & 36.55 \\
26 & 61.31 & 61.97 & 62.40 & 65.80 & 61.39 \\
27 & 13.63 & 12.97 & 12.52 & 13.29 & 12.98 \\
$24-O-a c e t y l$ & & - & & & \\
1 & - & - & 21.25 & & \\
2 & - & - & &
\end{tabular}

a Tested in $\mathrm{CD}_{3} \mathrm{OD}{ }^{\text {b }}$ tested in $\mathrm{C}_{5} \mathrm{D}_{5} \mathrm{~N}$.

Table 6. ${ }^{13} \mathrm{C}-\mathrm{NMR}$ data of the sugar portion of compounds $\mathbf{6 - 1 0 .}$

\begin{tabular}{cccccc}
\hline Sugars & \multicolumn{5}{c}{ Compounds } \\
\hline Ara $(p)$ & $\mathbf{6}^{\mathbf{b}}$ & $\mathbf{7}^{\mathbf{a}}$ & $\mathbf{8}^{\mathbf{a}}$ & $\mathbf{9}^{\mathbf{a}}$ & $\mathbf{1 0}^{\mathbf{a}}$ \\
\hline 1 & 101.31 & 101.61 & 101.53 & 101.64 & 101.26 \\
2 & 74.21 & 74.46 & 74.58 & 74.51 & 74.28 \\
3 & 85.32 & 85.37 & 85.42 & 71.21 & 85.32 \\
4 & 70.24 & 69.96 & 70.65 & 85.37 & 70.54 \\
5 & 67.58 & 67.18 & 67.17 & 67.04 & 67.08 \\
Rha & & & & & \\
1 & 102.02 & 101.47 & 101.69 & 101.47 & 101.71 \\
2 & 72.39 & 71.96 & 72.00 & 71.98 & 72.38 \\
3 & 80.40 & 80.46 & 80.48 & 80.46 & 72.12 \\
4 & 73.17 & 73.01 & 73.06 & 73.03 & 74.42 \\
5 & 70.13 & 69.80 & 69.84 & 69.81 & 69.87 \\
6 & 19.63 & 18.73 & 18.72 & 18.73 & 18.64 \\
Xyl & & & & & \\
1 & 107.16 & 106.50 & 106.54 & 106.52 & 106.49 \\
2 & 75.23 & 74.88 & 74.92 & 74.90 & 74.90 \\
3 & 78.95 & 78.05 & 78.09 & 78.05 & 70.99 \\
4 & 71.59 & 71.19 & 71.23 & 70.65 & 67.02 \\
5 & 67.58 & 67.02 & 67.04 & 68.18 & 101.26 \\
Api & & & & & 74.28 \\
1 & 112.31 & 112.13 & 112.18 & 112.16 & 70.54 \\
2 & 78.36 & 78.24 & 78.27 & 78.25 & 67.08 \\
3 & 80.83 & 80.48 & 80.48 & 80.48 & \\
4 & 75.73 & 75.16 & 75.18 & 65.59 & \\
5 & 66.17 & 65.56 & 65.59 & & \\
\hline
\end{tabular}

a Tested in $\mathrm{CD}_{3} \mathrm{OD} ;{ }^{\mathrm{b}}$ tested in $\mathrm{C}_{5} \mathrm{D}_{5} \mathrm{~N}$

The discovery of the new compounds 1-5 extend the diversity and complexity of the spirostane saponin family. The cytotoxicity of 1-5 was evaluated against human pancreatic adenocarcinoma PANC-1 and BxPC3 cell lines using the CCK8 method. Regrettably, none of compounds showed significant cytotoxicity (Table 7). 
Table 7. Cytotoxic activity of compounds $\mathbf{1 - 5}$ against human pancreatic cancer cells in vitro $\left(\mathrm{IC}_{50}, \mu \mathrm{M}\right)$.

\begin{tabular}{ccc}
\hline \multirow{2}{*}{ Compound } & \multicolumn{2}{c}{ Cytotoxic Activity $\left(\mathrm{IC}_{\mathbf{5 0}, \boldsymbol{\mu M} ; \text { Mean }} \pm \mathrm{SD}, \boldsymbol{n = 3}\right)$} \\
\cline { 2 - 3 } & PANC-1 & BxPC-3 \\
\hline $\mathbf{1}$ & $>80$ & $>80$ \\
$\mathbf{2}$ & $>80$ & $>80$ \\
$\mathbf{4}$ & $>80$ & $>80$ \\
$\mathbf{5}$ & $>80$ & $>80$ \\
Gemcitabine $^{\text {a }}$ & $>80$ & $>80$ \\
a Positive control. & $0.0927 \pm 0.0057$ & $0.0376 \pm 0.0031$ \\
\hline
\end{tabular}

${ }^{a}$ Positive control.

\section{Materials and Methods}

\subsection{General}

Optical rotations were measured on a Perkin-Elmer 241 MC digital polarimeter (German PerkinElmer Corporation, Boelingen, Germany). 1D and 2D-NMR spectral experiments were measured in CD3OD on a Bruker AVANCE-500 and a Bruksmer AVANCE-800 spectrometer (Bruker Corporation, Karlsruhe, Germany) with TMS as an internal standard. The IR spectra were recorded on a Shimadzu IRPrestige-21 spectrophotometer (Shimadzu Corporation, Tokyo, Japan). The ESI-MS and HR-ESI-MS spectra were carried out on a Waters Micromass Quattro mass spectrometer (Waters, Shanghai, China). Column chromatographies (CC) were operated on a Sephadex LH-20 (GE-Healthcare, Uppsala, Sweden), ODS silica gel (Lichroprep RP-18, 40-63 $\mu \mathrm{m}$, Merck Inc., Darmstadt, Germany), and silica gel H (10-40 $\mu \mathrm{m}$, Qingdao Marine Chemical Inc., Qingdao, China). The GC analysis was performed on an Agilent $6890 \mathrm{~N}$ apparatus using an HP-5 capillary column (30 m $\times 0.32 \mathrm{~mm}, 0.5 \mu \mathrm{m}$ ) and an FID detector with an initial temperature of $120^{\circ} \mathrm{C}$ for $2 \mathrm{~min}$ and then temperature programming to $280^{\circ} \mathrm{C}$ at the rate of $10^{\circ} \mathrm{C} / \mathrm{min}$. Standards for Dxylopyranose (D-Xyl), L-arabopyranose (L-Ara), and L-rhamnose (L-Rha) were purchased from Sigma Chemical Co. (St. Louis, MO, USA), and D-apiose (D-Api) was purchased from Herbest Bio-Tech Co. (St. Baoguo, Baoji, China).

\subsection{Plant Material}

The rhizomes of Paris mairei were collected from Lijiang, Yunnan Province, China, in September 2018 and identified by the corresponding author Haifeng Tang. The voucher sample (No. 20180903) was deposited in the Department of Chinese Materia Medica and Natural Medicines, School of Pharmacy, Air Force Medical University, Xi'an, China.

\subsection{Extraction and Isolation}

The dried rhizomes of Paris mairei $(1.0 \mathrm{~kg}$ ) were chopped and refluxed with $70 \%$ ethanol $(10.0 \mathrm{~L})$ thrice (each $2 \mathrm{~h}$ ). The ethanol solution was mixed and condensed with a vacuum rotary evaporator to receive a syrupy residue $(584.0 \mathrm{~g})$. The extraction was suspended in water $(3.0 \mathrm{~L})$ and extracted with same volume of petroleum ether and water saturated n-BuOH 3 times, successively. The water saturated in the n- $\mathrm{BuOH}$ layer was vacuum evaporated to give a gummy residue $(132.0 \mathrm{~g})$. The crude extraction was separated by silica gel column chromatography and eluted by gradient eluent of $\mathrm{CH}_{2} \mathrm{Cl}_{2}$ $\mathrm{MeOH}-\mathrm{H}_{2} \mathrm{O}$ (100:0:0, 50:1:0, 20:1:0, 8:1:0.1, 6:1:0.1, 8:2:0.2, 7:2.5:0.1, and 6.5:3.5:0.1) to offer 13 fractions (Fr.1-13) based on the TLC analysis. Fr.13 was separated by silica gel column chromatography and eluted by a gradient eluent of $\mathrm{CH}_{2} \mathrm{Cl}_{2}-\mathrm{MeOH}-\mathrm{H}_{2} \mathrm{O}$ (8:1:0.1, 8:2:0.2, 7:2.5:0.1, and 6:3:0.1) to get Fr.13-1 (1.1 g) and Fr.13-2 (830 mg). Fr.13-1 was eluted by $\mathrm{MeOH}$ on a Sephadex LH-20 to get rid of pigmentum and separated to Fr.13-1-1 (64 mg), Fr.13-1-2 (57 mg), and Fr-13-1-3 (145 mg) on ODS silica gel. Then, Fr.13-1-1 and Fr.13-1-3 were isolated by semi-preparative HPLC using $\mathrm{MeCN}^{-} \mathrm{H}_{2} \mathrm{O}(35: 65,40: 60)$ as the mobile phase at a flow rate of $8.0 \mathrm{~mL} / \mathrm{min}$ to afford compound $\mathbf{1}\left(9.1 \mathrm{mg}, \mathrm{t}_{R}=24.3 \mathrm{~min}\right)$ and $4(8.8 \mathrm{mg}$, $\mathrm{t}_{R}=48.6 \mathrm{~min}$ ), respectively. Fr.11 was eluted by MeOH on a Sephadex LH-20 to remove pigmentum to receive Fr.11-1 (4.2 g), Fr.11-2 (5.0 g), and Fr.11-3 (430 mg). Fr.11-2 was 
subjected to ODS silica gel and purified by a semi-preparative HPLC using $\mathrm{MeCN}-\mathrm{H}_{2} \mathrm{O}$ (50:50) as the mobile phase at a flow rate of $8.0 \mathrm{~mL} / \mathrm{min}$ to afford compound 3 (5.7 $\mathrm{mg}$, $\left.\mathrm{t}_{R}=44.1 \mathrm{~min}\right)$ and compound $7\left(7.6 \mathrm{mg}, \mathrm{t}_{R}=40.2 \mathrm{~min}\right)$. Fr.12 was eluted by $\mathrm{CH}_{2} \mathrm{Cl}_{2}-\mathrm{MeOH}$ (20:80) on a Sephadex LH-20 to remove pigmentum and subjected to ODS silica gel to obtain Fr.12-1 (125 mg) and Fr.12-2 (670 mg). Then, compound $2\left(26.7 \mathrm{mg}, \mathrm{t}_{R}=21.0 \mathrm{~min}\right)$ was offered by semi-preparative HPLC using $\mathrm{MeCN}^{-\mathrm{H}_{2} \mathrm{O}}(60: 40)$ as the mobile phase at a flow rate of $8.0 \mathrm{~mL} / \mathrm{min}$. Fr.9 was purified by $\mathrm{MeOH}$ on a Sephadex LH-20 and separated on ODS silica gel to obtain Fr.9-1 (231 mg), Fr.9-2 (102 mg), and Fr.9-3 (193 mg). The three collections were successively purified by semi-preparative $\mathrm{HPLC}$ using $\mathrm{MeCN}-\mathrm{H}_{2} \mathrm{O}$ (50:50, $40: 60,40: 60)$ as the mobile phase at a flow rate of $8.0 \mathrm{~mL} / \mathrm{min}$ to obtain compounds 5 $\left(11.5 \mathrm{mg}, \mathrm{t}_{R}=35.3 \mathrm{~min}\right), 8\left(5.5 \mathrm{mg}, \mathrm{t}_{R}=38.4 \mathrm{~min}\right)$, and $\mathbf{9}\left(4.6 \mathrm{mg}, \mathrm{t}_{R}=28.7 \mathrm{~min}\right)$. Fr.10 was eluted by $\mathrm{CH}_{2} \mathrm{Cl}_{2}-\mathrm{MeOH}$ (50:50) on a Sephadex $\mathrm{LH}-20$ to remove pigmentum and subjected to ODS silica gel to obtain Fr.10-1 (75 mg) and Fr.10-2 (100 mg). Fr.10-1 and Fr.10-2 were isolated by semi-preparative HPLC using $\mathrm{MeCN}_{-} \mathrm{H}_{2} \mathrm{O}(75: 25)$ as the mobile phase at a flow rate of $8.0 \mathrm{~mL} / \mathrm{min}$ to afford compounds $10\left(7.5 \mathrm{mg}, \mathrm{t}_{R}=18.3 \mathrm{~min}\right)$ and $\mathbf{6}$ $\left(24.4 \mathrm{mg}, \mathrm{t}_{R}=21.6 \mathrm{~min}\right)$, respectively. The purity of all compounds was assessed by HPLC as being more than $95 \%$.

\subsection{Compound Characterization Data}

Pamaiosides A (1): white amorphous solid, $[\alpha]_{22 \mathrm{D}}-95.0$ (c 0.05, MeOH); IR (KBr) $v_{\max }\left(\mathrm{cm}^{-1}\right): 3420,2930,1080,990$, and 840; positive ESI-MS $\mathrm{m} / z 995.13[\mathrm{M}+\mathrm{Na}]^{+}$, negative ESI-MS m/z 971.28 [M - H] $]^{-}$; positive HR-ESI-MS m/z $995.4824[\mathrm{M}+\mathrm{Na}]^{+}$(calculated for $^{2}$ $\mathrm{C}_{48} \mathrm{H}_{76} \mathrm{O}_{20} \mathrm{Na}$, 995.4828); ${ }^{1} \mathrm{H}-\mathrm{NMR}\left(800 \mathrm{MHz}, \mathrm{CD}_{3} \mathrm{OD}\right)$ and ${ }^{13} \mathrm{C}-\mathrm{NMR}\left(201 \mathrm{MHz}, \mathrm{CD}_{3} \mathrm{OD}\right)$ data, see Table 1.

Pamaiosides B (2): white amorphous solid, $[\alpha]_{22 \mathrm{D}}-98.2$ (c 0.06, MeOH); IR (KBr) $v_{\max }$ $\left(\mathrm{cm}^{-1}\right)$ : 3422, 2932, 1078, 988, and 840; positive ESI-MS m/z $1043.49[\mathrm{M}+\mathrm{Na}]^{+}$; positive HR-ESI-MS $m / z 1043.4677\left[\mathrm{M}+\mathrm{Na}{ }^{+}\right.$(calculated for $1043.4675 \mathrm{C}_{48} \mathrm{H}_{76} \mathrm{O}_{23} \mathrm{Na}$ ); ${ }^{1} \mathrm{H}-\mathrm{NMR}$ $\left(500 \mathrm{MHz}, \mathrm{CD}_{3} \mathrm{OD}\right)$ and ${ }^{13} \mathrm{C}-\mathrm{NMR}\left(125 \mathrm{MHz}, \mathrm{CD}_{3} \mathrm{OD}\right)$ data, see Table 2.

Pamaiosides C (3): White amorphous solid, $[\alpha]_{22 \mathrm{D}}-105.2$ (c 0.10, $\mathrm{MeOH}$; IR (KBr) $\gamma_{\max }\left(\mathrm{cm}^{-1}\right): 3420,2930,1668,1078,989$, and 842; positive ESI-MS $\mathrm{m} / \mathrm{z} 993.48[\mathrm{M}+\mathrm{Na}]^{+}$; negative ESI-MS $m / z 969.28\left[\mathrm{M}-\mathrm{H}^{-}{ }^{-}\right.$; positive HR-ESI-MS $\mathrm{m} / z$ $993.3932[\mathrm{M}+\mathrm{Na}]^{+}$(calculated for $\left.993.3943 \mathrm{C}_{46} \mathrm{H}_{66} \mathrm{O}_{22} \mathrm{Na}\right) ;{ }^{1} \mathrm{H}-\mathrm{NMR}\left(800 \mathrm{MHz}, \mathrm{CD}_{3} \mathrm{OD}\right)$ and ${ }^{13} \mathrm{C}-\mathrm{NMR}(201 \mathrm{MHz}$, $\mathrm{CD}_{3} \mathrm{OD}$ ) data, see Table 3.

Pamaiosides D (4): White amorphous solid, $[\alpha]_{22 \mathrm{D}}-110.0$ (c 0.10, MeOH); IR (KBr) $v_{\max }\left(\mathrm{cm}^{-1}\right): 3432,2922,1664,1080,990,837$; Positive ESI-MS m/z $993.18[\mathrm{M}+\mathrm{Na}]^{+}$; Negative ESI-MS $m / z$ 969.36 [M - H] ${ }^{-}$; Positive HR-ESI-MS $m / z 993.3969[\mathrm{M}+\mathrm{Na}]^{+}$ (calcd. for $\mathrm{C}_{46} \mathrm{H}_{66} \mathrm{O}_{22} \mathrm{Na}$, 993.3943); ${ }^{1} \mathrm{H}-\mathrm{NMR}\left(800 \mathrm{MHz}, \mathrm{CD}_{3} \mathrm{OD}\right)$ and ${ }^{13} \mathrm{C}-\mathrm{NMR}(201 \mathrm{MHz}$, $\left.\mathrm{CD}_{3} \mathrm{OD}\right)$ data, see Table 4.

Pamaiosides E (5): White amorphous solid, $[\alpha]_{22 \mathrm{D}}-109.0$ (c $\left.0.15, \mathrm{MeOH}\right) ; \mathrm{IR}(\mathrm{KBr})$ $v_{\max }\left(\mathrm{cm}^{-1}\right): 3430,2925,1080,990$, and 840; positive ESI-MS $\mathrm{m} / \mathrm{z} 935.39[\mathrm{M}+\mathrm{Na}]^{+}$; positive HR-ESI-MS $m / z$ 935.3892 [M + Na] ${ }^{+}$(calculated for $\mathrm{C}_{44} \mathrm{H}_{64} \mathrm{O}_{20} \mathrm{Na}$, 935.3889); ${ }^{1} \mathrm{H}-\mathrm{NMR}$ $\left(500 \mathrm{MHz}, \mathrm{CD}_{3} \mathrm{OD}\right)$ and ${ }^{13} \mathrm{C}-\mathrm{NMR}(125 \mathrm{MHz}, \mathrm{CD} 3 \mathrm{OD})$ data, see Table 5.

All the NMR (1D and 2D) and MS spectra of compounds 1-5 could be found in Supplementary Materials (Figures S1-S55).

\subsection{Acid Hydrolysis and GC Analysis of the Sugar Moieties in Compounds 1-5}

The assay was performed according to the procedure of Qiang F., et al. [28] with slight modifications, using $\mathrm{N}$-(trimethylsilyl)imidazole as derivatization substrate. Compounds 1-5 (each $2 \mathrm{mg}$ ) were mixed with $2 \mathrm{~mol} / \mathrm{L} \mathrm{CF}_{3} \mathrm{COOH}(2 \mathrm{~mL})$ and heated in a sealed tube at $110{ }^{\circ} \mathrm{C}$ for $8 \mathrm{~h}$. Distilled water $(20 \mathrm{~mL})$ was added when the reaction was over and extracted with EtOAc $(20 \mathrm{~mL})$ three times. The aqueous layer was concentrated in vacuo by repeated mixing with methanol until the solvent was completely evaporated. The residue was dissolved in a $1 \mathrm{~mL}$ pyridine solution of $2 \mathrm{mg} / \mathrm{L}$ of L-cysteine methyl ester hydrochloride. After warming at $60^{\circ} \mathrm{C}$ for $1 \mathrm{~h}$, the solvent was evaporated under 
$\mathrm{N}_{2}$ protection. The reaction products were dissolved in the mixed solution of $0.2 \mathrm{~mL}$ $\mathrm{N}$-(trimethylsilyl)imidazole and $2 \mathrm{~mL}$ anhydrous pyridine, and the mixture was warmed at $60{ }^{\circ} \mathrm{C}$ for another $1 \mathrm{~h}$. Then, the solvent was evaporated under $\mathrm{N}_{2}$ protection. The residue was suspended in cyclohexane and water, the cyclohexane layer was the trimethylsilyl ether derivatives of monosaccharide. The mixture was filtered through a $0.45 \mu \mathrm{m}$ membrane to remove the precipitate and analyzed by GC. Separations were carried out on an HP- 5 capillary column $(30 \mathrm{~m} \times 0.32 \mathrm{~mm}, 0.5 \mu \mathrm{m})$. Highly pure $\mathrm{N}_{2}$ was used as a carrier gas $\left(1.0 \mathrm{~mL} / \mathrm{min}\right.$ flow rate), and the FID detector operated at $250{ }^{\circ} \mathrm{C}$ (column temperature $250^{\circ} \mathrm{C}$ ). The carbohydrates were determined by comparing the retention times with standard trimethylsilyl ether derivatives prepared from authentic sugars using the same procedure performed for the sample. Retention times for authentic sugars after being derivatized were $11.23 \mathrm{~min}$ (D-Api), $12.20 \mathrm{~min}$ (L-Ara), $13.34 \mathrm{~min}$ (D-Xyl), and $14.48 \mathrm{~min}$ (L-Rha), respectively.

\subsection{Cytotoxicity Assay for Compounds 1-5}

The human pancreatic adenocarcinoma PANC-1 and BxPC3 cell lines were purchased from the Cell Bank of the Chinese Academy of Science (Shanghai, China) and cultured in DMEM (Corning, Beijing, China) supplemented with 10\% FBS (Sigma, Shanghai, China) and $1 \%$ Penicillin-Streptomycin (Sigma, Shanghai, China) at $37^{\circ} \mathrm{C}$ with $5 \% \mathrm{CO}_{2}$. In the exponential phase of the growth, cells were plated onto 96-well plates at a concentration of 8000 cells /well for $24 \mathrm{~h}$. Compounds 1-5 were prepared to various concentrations (80, $40,20,10,5,2.5,1.25$, and $0.625 \mu \mathrm{M}$ in medium containing less than $0.1 \% \mathrm{DMSO}$ ) and incubated in 96-well plates (each concentration in six-fold wells) for $72 \mathrm{~h}$. Gemcitabine (Gem, Meilunbio, $\geq 98 \%$, Dalian, China) was offered as the positive control. Cell viability was determined according to reported assay methods using the commercial CCK8 kit (Elabscience, Wuhan, China) [29]. The optical density (OD) of each well was measured with an AMR-100 microplate reader at $450 \mathrm{~nm}$ (Allsheng Corporation, Hangzhou, China). Cytotoxicity emerged as the value of the drug concentration at the inhibition of cell growth by $50 \%\left(\mathrm{IC}_{50}\right)$.

\section{Conclusions}

This study afforded 10 compounds from the rhizomes of Paris mairei, including five new spirostane saponins. None of the new compounds exhibited cytotoxicity against PANC-1 and BxPC3 pancreatic cell lines, implying that the polyglucosides at 1-hydroxy in spirostane saponins may significantly decreased the activities of antitumor.

Supplementary Materials: The following are available online, Figures S1-S55: NMR (1D and 2D) and MS spectra of compounds 1-5.

Author Contributions: Conceptualization, H.T. and B.-L.Z.; NMR data, M.W.; acid hydrolysis and GC analysis, P.Q.; cytotoxicity evaluation, H.H.; isolation, structural identification, and writingoriginal draft preparation, Y.L. (Yang Liu); writing—review and editing, Y.L. (Yunyang Lu). All authors have read and agreed to the published version of the manuscript.

Funding: The research work was financially supported by the National Natural Science Foundation of China (No. 81973192 and No.81903862) and the Shaanxi Province Key Research and Development Projects of China (No.2021ZDLSF04-07 and No. 2020SF-311).

Institutional Review Board Statement: Not applicable.

Informed Consent Statement: Not applicable.

Data Availability Statement: The data used to support the findings of this study are available from the corresponding author upon request.

Conflicts of Interest: The authors declare no conflict of interest.

Sample Availability: Samples of the compounds 6-10 are available from the authors. 


\section{References}

1. Ding, Y.G.; Zhao, Y.L.; Zhang, J.; Zuo, Z.T.; Zhang, Q.Z.; Wang, Y.Z. The traditional uses, phytochemistry, and pharmacological properties of Paris, L. (Liliaceae): A review. J. Ethnopharmacol. 2021, 278, 114293. [CrossRef] [PubMed]

2. Liu, Y.; Wang, M.; Liu, K.; Qiu, P.C.; Zhang, S.; Lu, Y.Y.; Tang, N.; Tang, H.F. New steroidal saponins from the rhizomes of Paris vietnamensis and their cytotoxicity. Molecules 2018, 23, 588. [CrossRef] [PubMed]

3. Dong, R.Z.; Zhang, Z.W.; Zhou, Y.M.; Guo, J.M. The effect of polyphyllin I on gastric cancer-associated fibroblasts. J. Clin. Oncol. 2018, 24, 336-340. [CrossRef]

4. Luo, Q.; Yang, D.; Qi, Q.; Chen, B.; Liu, W.; Shi, L.; Xia, Y.; Tang, L.; Fang, J.; Ou, Y.; et al. Role of the Death Receptor and Endoplasmic Reticulum Stress Signaling Pathways in Polyphyllin I-Regulated Apoptosis of Human Hepatocellular Carcinoma HepG2 Cells. BioMed Res. Int. 2018, 2018, 5241941. [CrossRef] [PubMed]

5. Yan, T.; Hu, G.; Wang, A.; Sun, X.; Yu, X.; Jia, J. Paris saponin VII induces cell cycle arrest and apoptosis by regulating Akt/MAPK pathway and inhibition of P-glycoprotein in K562/ADR cells. Phytother. Res. 2018, 32, 898-907. [CrossRef]

6. Zhang, C.; Li, C.; Jia, X.; Wang, K.; Tu, Y.; Wang, R.; Liu, K.; Lu, T.; He, C. In Vitro and In Vivo Anti-Inflammatory Effects of Polyphyllin VII through Downregulating MAPK and NF-kB Pathways. Molecules 2019, 24, 875. [CrossRef]

7. Qin, X.J.; Sun, D.J.; Ni, W.; Chen, C.X.; Hua, Y.; He, L.; Liu, H.Y. Steroidal saponins with antimicrobial activity from stems and leaves of Paris polyphylla var. yunnanensis. Steroids 2012, 77, 1242-1248. [CrossRef]

8. Sun, C.L.; Ni, W.; Yan, H.; Liu, Z.H.; Yang, L.; Si, Y.A.; Hua, Y.; Chen, C.X.; He, L.; Zhao, J.H.; et al. Steroidal saponins with induced platelet aggregation activity from the aerial parts of Paris verticillata. Steroids 2014, 92, 90-95. [CrossRef]

9. Yu, J.; Deng, H.; Xu, Z. Targeting macrophage priming by polyphyllin VII triggers anti-tumor immunity via STING-governed cytotoxic T-cell infiltration in lung cancer. Sci. Rep. 2020, 10, 21360. [CrossRef]

10. Pei, Y.; Zhang, Q.; Wang, Y. Application of authentication evaluation techniques of ethnobotanical medicinal plant genus Paris: A Review. Crit. Rev. Anal. Chem. 2020, 50, 405-423. [CrossRef]

11. Chen, P.Y.; Chen, C.H.; Kuo, C.C.; Lee, T.H.; Kuo, Y.H.; Lee, C.K. Cytotoxic steroidal saponins from Agave sisalana. Planta Med. 2011, 77, 929-933. [CrossRef]

12. Liu, H.; Chou, G.X.; Wang, J.M.; Ji, L.L.; Wang, Z.T. Steroidal saponins from the rhizomes of Dioscorea bulbifera and their cytotoxic activity. Planta Med. 2011, 77, 845-848. [CrossRef] [PubMed]

13. Akihiko, T.A.; Mutsumi, T.A.; Junich, K.A.; Kawase, M.; Miyamae, H.; Yoza, K.; Takasaki, A.; Nagamura, Y.; Saito, S. Spirostanols obtained by cyclization of pseudosaponin derivatives and comparison of anti-platelet agglutination activities of spirostanol glycosides. Eur. J. Med. Chem. 2000, 35, 511-527. [CrossRef]

14. Minh, C.V.; Dat, N.T.; Dang, N.H.; Nam, N.H.; Ban, N.K.; Tuyen, N.V.; Huong, L.M.; Huong, T.T.; Kiem, P.V. Unusual 22Sspirostane steroids from Dracaena cambodiana. Nat. Prod. Commun. 2009, 4, 1197-1200. [CrossRef]

15. Agrawal, P.K.; Jain, D.C.; Gupta, R.K.; Thakur, R.S. Carbon-13 NMR spectroscopy of steroidal sapogenins and steroidal saponins. Phytochemistry 1985, 24, 2479-2496. [CrossRef]

16. Agrawal, P.K. Assigning stereodiversity of the 27-Me group of furostane-type steroidal saponins via NMR chemical shifts. Steroids 2005, 70, 715-724. [CrossRef]

17. Seigler, D.S.; Pauli, G.F.; Nahrstedt, A.; Leen, R. Cyanogenic allosides and glucosides from Passiflora edulis and Carica papaya. Phytochemistry 2002, 60, 873-882. [CrossRef]

18. Lu, Y.Y.; Wang, M.C.; Liu, K.; Zhang, W.; Liu, Y.; Tang, H.F. Two new polyhydroxylated steroidal glycosides from the starfish Culcita novaeguineae. Nat. Prod. Res. 2020, 18, 1-7. [CrossRef]

19. Qin, X.J.; Si, Y.A.; Chen, Y.; Liu, H.; Ni, W.; Yan, H.; Shu, T.; Ji, Y.H.; Liu, H.Y. Cytotoxic steroidal saponins from Trillium kamtschaticum. Bioorg. Med. Chem. Lett. 2017, 27, 2267-2273. [CrossRef] [PubMed]

20. Snyder, J.R.; Serianni, A.S. DL-apiose substituted with stable isotopes: Synthesis, n.m.r.-spectral analysis, and furanose anomerization. Carbohydr. Res. 1987, 166, 85-99. [CrossRef]

21. Liu, Y.; Tian, X.R.; Hua, D.; Cheng, G.; Wang, K.; Zhang, L.; Tang, H.F.; Wang, M.C. New steroidal saponins from the rhizomes of Paris delavayi and their cytotoxicity. Fitoterapia 2016, 111, 130-137. [CrossRef]

22. Meng, C.W.; Peng, C.; Zhou, Q.M.; Yang, H.; Guo, L.; Xiong, L. Spirostanols from the roots and rhizomes of Trillium tschonoskii. Phytochem. Lett. 2015, 14, 134-137. [CrossRef]

23. Nohara, T.; Komori, T.; Kawasaki, T. Steroid saponins and sapogenins of underground parts of Trillium kamtschaticum pall. III. on the structure of a novel type of steroid glycoside, Trillenoside A, an 18-norspirostanol oligoside. Chem. Pharm. Bull. 2008, 28, 1437-1448. [CrossRef]

24. Ono, M.; Sugita, F.; Shigematsu, S.; Takamura, C.; Yoshimitsu, H.; Miyashita, H.; Ikeda, T.; Nohara, T. Three new steroid glycosides from the underground parts of Trillium kamtschaticum. Chem. Pharm. Bull. 2007, 55, 1093-1096. [CrossRef]

25. Zhang, Z.L.; Cai, M.T.; Zuo, Y.M.; Wang, Y.Y. Studies on chemical constituents in the fruits of Trillium tschonoskii Maxim. Lishizhen Med. Mater. Med. Res. 2014, 25, 541-543.

26. Jiangxi University of Traditional Chinese Medicine. Application of Trillium tschonoskii Steroidal Saponin Extract and Steroidal Saponins therein in Preparing Drugs for Treating Ulcerative Colitis. CN201811047138.1, 8 September 2018. (Application date).

27. Chai, J.; Song, X.; Wang, X.; Mei, Q.; Li, Z.; Cui, J.; Tang, Z.; Yue, Z. Two new compounds from the roots and rhizomes of Trillium tschonoskii. Phytochem. Lett. 2014, 10, 113-117. [CrossRef] 
28. Fu, Q.; Zan, K.; Zhao, M.; Zhou, S.; Shi, S.; Jiang, Y.; Tu, P. Triterpene saponins from Clematis chinensis and their potential anti-inflammatory activity. J. Nat. Prod. 2010, 73, 1234-1239. [CrossRef] [PubMed]

29. Tian, D.Y.; Teng, X.; Jin, S.; Chen, Y.; Xue, H.; Xiao, L.; Wu, Y. Endogenous hydrogen sulfide improves vascular remodeling through PPARס $/ \mathrm{SOCS}_{3}$ signaling. J. Adv. Res. 2020, 27, 115-125. [CrossRef] 